\title{
The catalytic regio- and stereoselective synthesis of 1,6-diazabicy-
}

\section{clo[4.3.0]nonane-2,7-diones}

\author{
Arthur Lebrêne, ${ }^{\mathrm{a}}$ Thomas Martzel, ${ }^{\mathrm{a}}$ Laura Gouriou, ${ }^{\text {a† }}$ Morgane Sanselme, ${ }^{\mathrm{b}}$ Vincent Levacher, ${ }^{\text {a Syl- }}$ \\ vain Oudeyer, ${ }^{a}$ Carlos Afonso, ${ }^{\text {a† }}$ Corinne Loutelier-Bourhis ${ }^{\text {a†* }}$ and Jean-François Brière ${ }^{a *}$
}

Supporting Information Placeholder

\begin{abstract}
A straightforward synthesis of original 1,6-diazabicyclo[4.3.0]nonane-
2,7-diones was achieved through a DBU-organocatalyzed multicomponent

Knoevenagel-aza-Michael-Cyclocondensation (KMC) reaction which takes ad-

vantage of an unprecedented highly regio- and diastereoselective conjugate addi-

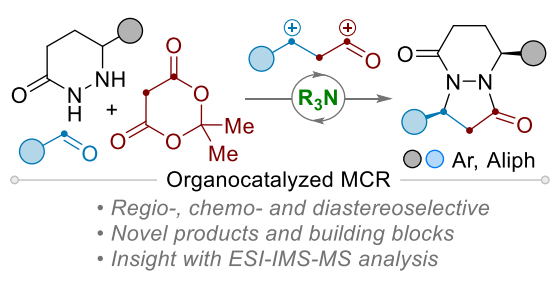

tion of pyridazinones to alkylidene Meldrum's acid intermediates. The key reactive intermediates of this complex process were analyzed by means of electrospray ionization mass spectrometry coupled to ion mobility spectrometry (ESI-IMS-MS) allowing to valid the proposed mechanism.
\end{abstract}

\section{INTRODUCTION}

The development of new routes to synthesize small chiral azaarenes has become an important exercise in drug development. ${ }^{1}$ These $\mathrm{Csp}^{3}$ - rich heterocycles proved to be versatile platforms e.g., for the elaboration of novel drug candidates or to populate compound libraries useful for the increasingly popular fragment-based drug discovery (FBDD). ${ }^{2}$ By exploring the 3D-chemical space, it has been shown that $\mathrm{sp}^{3}$-rich compounds could lead to better selectivity towards the bio-targets while decreasing the toxicity issues. $^{3}$ In that context, the substituted diazabicyclo[4.3.0]nonanone derivatives $\mathbf{1}$ and $\mathbf{2}$ are versatile fused bicyclic structures (Scheme 1), also encountered within bioactive molecules. ${ }^{4,5,6}$ Amongst the most straightforward stereoselective syntheses of these types of molecules, the transformation of chiral azomethine imines (AI), accessible from the corresponding aldehydes $\left(\mathrm{R}^{1} \mathrm{CHO}\right)$ and cyclic hydrazines ( $\left.\mathrm{R}^{2} \mathrm{NH}-\mathrm{NHR}\right)$, stands out (Scheme $\left.1 \mathrm{~A}\right) .^{4}$ The diastereoselective $(3+3)$ cycloadditions of readily available chiral Dorn-Otto type five-membered ring azomethine imines AI-a have recently emerged to give 1,6-diazabicyclo[4.3.0]nonan-7-ones $1 .^{5,6}$ On the other hand, Husson, Micouin and colleagues reported on an original $(3+2)$ cycloaddition allowing the construction of the five-membered 
ring moiety of the obtained isomeric 1,6-diaza-3-oxobicyclo[4.3.0]nonan-2-one product 2 , from the corresponding and much less explored six-membered ring derived dipoles AI-b. ${ }^{7}$

In quest of opening the topology space of these valuable diazabicyclo[4.3.0]nonanone derivatives, we tackled an alternative disconnection based on the regioselective addition of pyridazinones $\mathbf{5}$ to a three-carbon unit like $\mathbf{6}$ to form 1,6-diazabicyclo[4.3.0]nonane-2,7-diones 7 (Scheme 1B). During preliminary investigations, we discovered that chiral hydroxylamines and pyrazolidinones, as aza-bisnucleophiles, ${ }^{8}$ led to a novel domino aza-Michael-cyclocondensation sequence to the highly reactive and in-situ formed alkylidene Meldrum's acids 6 upon catalytic conditions. ${ }^{9}$ However, the application of this process to 6-membered ring pyridazinones $\mathbf{5}$ is not obvious and raised several questions concerning (1) the reactivity issue as long as the regioselective aza-Michael of these valuable cyclic hydrazines $\mathbf{5}$ remains largely unexplored. Next (2), the preliminary investigation with the 5-phenyl-pyrazolidinone (5-membered ring) showed that the diastereoselectivity outcome strongly depends on the nature of the aldehyde. ${ }^{8 a}$ Nonetheless, through the anticipated formal $(3+2)$ annulation between pyridazinones $\mathbf{5}$ and $\mathbf{6}$, contrarywise to the azomethine imine-based sequence (Scheme $1 \mathrm{~A}$ versus $1 \mathrm{~B}$ ), the $\mathrm{R}^{1}$ and $\mathrm{R}^{2}$ functional groups of the thus obtained bicyclic structure 7 will be apart to each other, and the two carbonyls lying at opposite position, which has been rarely described in the literature. ${ }^{10}$ Then, this sequence would eventually provide original products 7 with potentially unique properties and functionalization opportunities.

\section{Scheme 1. Known synthesis of 1,6-diazabicy-} clo[4.3.0]nonanones (A) and new development (B)

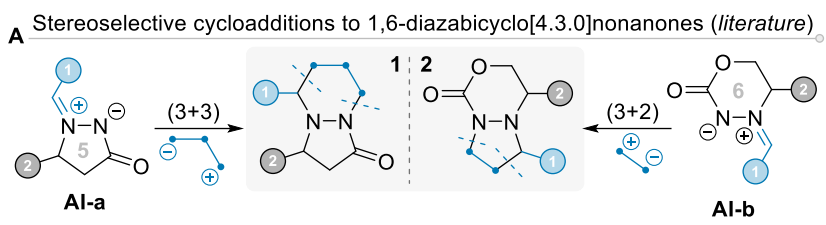

B $\quad(3+2)$ annulation towards novel 1,6-diazabicyclo[4.3.0]nonane-2,7-diones

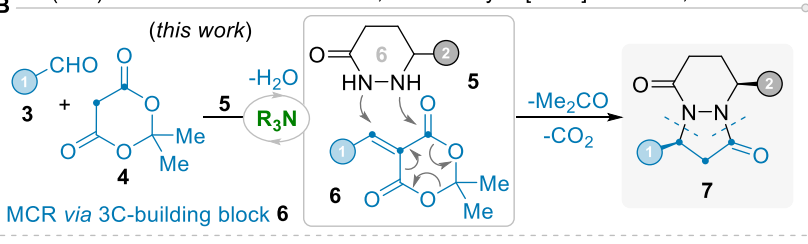

- Organocatalyzed MCR $(3+4+5) \cdot$ Regio- and diastereoselective $\cdot$ Novel products

Nevertheless, the orchestration of a versatile process, in an efficient multicomponent fashion, ${ }^{11}$ requires the selective formation and reaction of the alkylidene Meldrum's acid intermediate 6 (from aldehyde $3+4$ ) in the presence of pyridazinone 5, which might be in competition with the azomethine imine species, akin to AI-b, resulting from the condensation of $\mathbf{5}$ and $\mathbf{3}$. Notwithstanding these challenges, we are pleased to report that the chemoselective multicomponent Knoevenagel-aza-Michael-Cyclocondensation (KMC) reaction takes place under organocatalytic conditions and leads to the highly stereoselective synthesis of novel 1,6-diazabicyclo[4.3.0]nonane-2,7-diones 7. Importantly, an ESI-IMS-MS study allowed to get insight into the formation of key intermediates of this otherwise complex sequence.

\section{RESULTS AND DISCUSSION}

Table 1. Proof of concept and optimization 


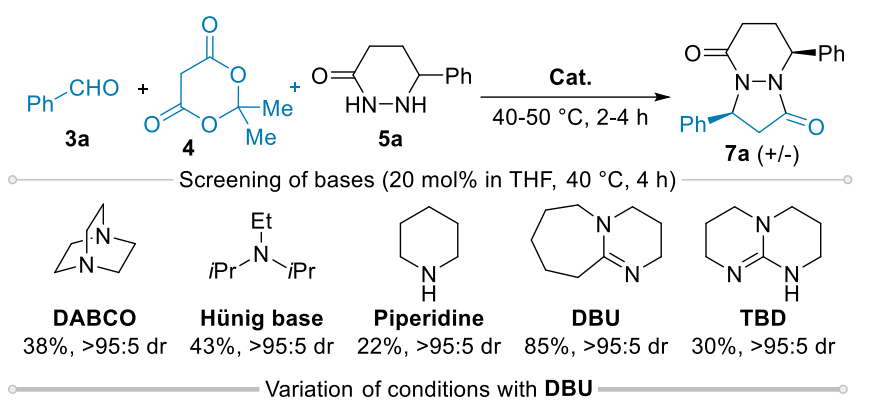

\begin{tabular}{|c|c|c|c|c|c|}
\hline Entry & $\begin{array}{l}\text { DBU } \\
\text { (mol\%) }\end{array}$ & Solvent & time/temp & $\begin{array}{l}\text { Yield } \\
(\%)^{a}\end{array}$ & $\mathrm{dr}^{b}$ \\
\hline 1 & 20 & $\mathrm{PhMe}$ & $2 \mathrm{~h} / 40^{\circ} \mathrm{C}$ & 7 & - \\
\hline 2 & 20 & $\mathrm{CH}_{2} \mathrm{Cl}_{2}$ & $2 \mathrm{~h} / 40^{\circ} \mathrm{C}$ & 8 & - \\
\hline 3 & 20 & AcOEt & $2 \mathrm{~h} / 40^{\circ} \mathrm{C}$ & 33 & $>95: 5$ \\
\hline 4 & 20 & $\mathrm{MeCN}$ & $2 \mathrm{~h} / 40^{\circ} \mathrm{C}$ & 14 & $>95: 5$ \\
\hline 5 & 20 & THF & $2 \mathrm{~h} / 40^{\circ} \mathrm{C}$ & 40 & $>95: 5$ \\
\hline 6 & 20 & THF & $4 \mathrm{~h} / 40^{\circ} \mathrm{C}$ & 85 & $>95: 5$ \\
\hline 7 & 10 & THF & $4 \mathrm{~h} / 40^{\circ} \mathrm{C}$ & 45 & $>95: 5$ \\
\hline 8 & 10 & THF & $4 \mathrm{~h} / 50^{\circ} \mathrm{C}$ & $88(83)^{c}$ & $>95: 5$ \\
\hline 9 & - & THF & $4 \mathrm{~h} / 50^{\circ} \mathrm{C}$ & $0(27)^{c}$ & - \\
\hline
\end{tabular}

Reaction performed at $0.1 \mathrm{M}$ on $0.2 \mathrm{mmol}$ scale with 1 equivalent of each component. ${ }^{a} \mathrm{NMR}$ yield determined by an internal standard. ${ }^{b}$ Diastereoisomeric ratio determined by ${ }^{1} \mathrm{H}$ NMR on the crude. ${ }^{c}$ The reaction was performed from pre-formed alkylidene Meldrum's acid 6a.

The tetrahydropyridazinones $\mathbf{5}$ are usually constructed by a linear condensation/reduction sequence from the keto-esters. We found that the addition of Grignard or organolithium reagents to the readily available 4,5-dihydropyridazin-3(2H)-one gave rise to a straightforward formation of various tetrahydropyridazinones $\mathbf{5}$ albeit in moderate yields (see experimental section). ${ }^{12}$ With this type of precursor in hands, the multicomponent reaction (MCR) was investigated by mixing pyridazinone 5a, benzaldehyde 3a and Meldrum's acid 4 (1 equivalent of each). We found that $20 \mathrm{~mol} \%$ of DABCO in THF for 4 hours at $40{ }^{\circ} \mathrm{C}$ gave the corresponding diazabicyclononanedione $\mathbf{7 a}$ in $38 \%$ NMR yield (Table 1-Screening of bases, see SI for further details). To our delight, essentially one diastereoisomer was obtained ( $>95: 5 \mathrm{dr}$ ) and, with regard to a series of X-Ray analyses (vide infra), the two phenyl moieties are likely lying cis to each other. The nature of the Brønsted base strongly influenced the efficiency of the reaction (Table 1), and the higher the $\mathrm{p} K_{\mathrm{aH}}$ (from DABCO, Hünig, piperidine bases and see SI other examples) the better the conversion with 1,8diazabicyclo[5.4.0] undec-7-ene (DBU) giving the best yield of $85 \%$ in only 4 hours. However, a plateau was reach as long as stronger guanidine bases, such as TBD, led to a lower yield of $30 \%$. As seen in entries 1-5, a faster MCR took place in THF as solvent and the organocatalyst loading could be decreased to $10 \mathrm{~mol} \%$ (entries 6-7), although a reaction temperature of $50^{\circ} \mathrm{C}$ was required to reach a complete conversion into $\mathbf{7 a}$ in an excellent $88 \%$ yield (entry 8). Despite no product formation was observed without DBU, $27 \%$ of diazabicyclononanedione 7a was obtained by starting directly from alkylidene Meldrum's acid 6a (entry 9), showing the likely formation of this intermediate $\mathbf{6 a}$ during the MCR (see also entry 8) and the role of the base on several stages of the catalytic cycle. During this optimization, the accumulation of an intermediate (vide infra) was observed at $40^{\circ} \mathrm{C}$, which indeed completely disappeared at $50{ }^{\circ} \mathrm{C}$; no trace of the regioisomer (from the azomethine imine intermediate) was detected on the crude product by ${ }^{1} \mathrm{H}$ NMR. 
To our delight, this MCR could be carried out with phenyl-pyridazinone 5a to furnish the corresponding product 7a as a single diastereoisomer in $81 \%$ isolated yield after column chromatography and $73 \%$ yield on $1 \mathrm{mmol}$ scale (Scheme 2 - variation of hydrazines). Furthermore, preliminary investigations showed that diazabicyclononanedione 7a could be obtained with $>99 \%$ enantiomeric excess from an enantiopure sample of pyridazinone 5a (see ES and SI).

\section{Scheme 2. Scope and limitation}
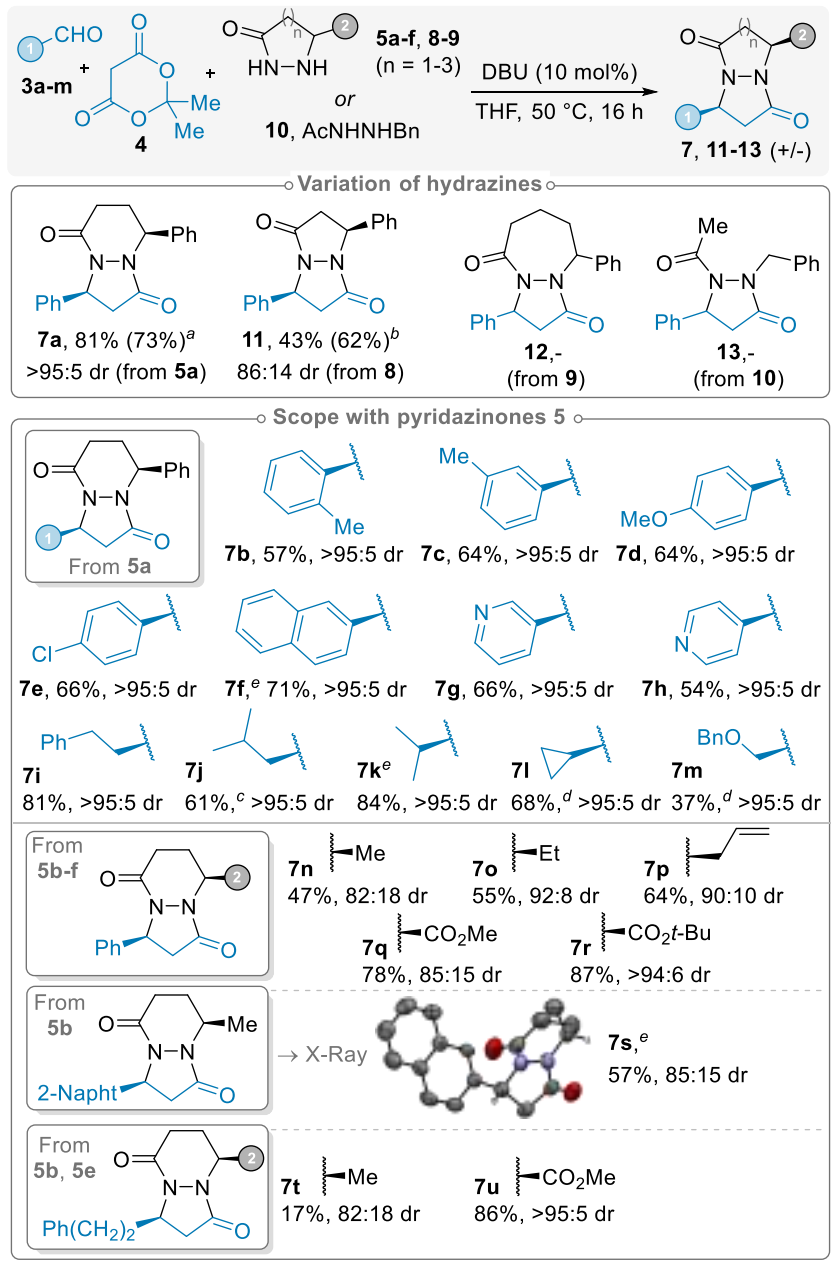

Reaction performed with pyridazinone 5 or analogues 8-10, aldehydes 3 ( 0.3 mmol, 1 equiv of each) and Meldrum's acid 4 (1.3 equiv.) in THF ( $0.1 \mathrm{M})$ at $50^{\circ} \mathrm{C}$ for $16 \mathrm{~h}$; isolated yields (\%) of the major diastereoisomer after column chromatography and $\mathrm{dr}$ determined by ${ }^{1} \mathrm{H}$ NMR on the crude product.
${ }^{a}$ Performed on $1 \mathrm{mmol}$ scale. ${ }^{b}$ Yield of both diastereoisomers. ${ }^{c} 30$ hours of reaction. ${ }^{d} 24$ hours of reaction. ${ }^{e}$ Structures determined by X-Ray diffraction analyses.

For a sake of comparison with a previous observation, ${ }^{8 a}$ these conditions could be applied to pyrazolidinone 8 to yield 11 in $62 \%$ but in a mixture of hardly separable diastereoisomers (86:14 dr). Furthermore, neither the sevenmembered ring hydrazine 9 nor the non-cyclic hydrazine 10 led to any pyrazolidinone derivatives $\mathbf{1 2 - 1 3}$ formation. These results show the specific reactivity and selectivity of pyridazinone nucleophiles in the multicomponent KMC reaction. Then, we explored the scope of this catalyzed MCR giving rise to the formation of novel 1,6-diazabicyclo[4.3.0]nonane-2,7-diones 7 (Scheme 2-Scope). Starting from pyridazinone $\mathbf{5 a}$, a series of bicyclic heterocycles $\mathbf{7 b}$ 7h could be easily elaborated with various aromatic aldehydes $\mathbf{3 b - 3 f}$ and heteroaromatic aldehydes $\mathbf{3 g - 3 h}$ with isolated yields ranging from $54 \%$ to $71 \%$. Aliphatic linear (3i3j), branched (3k) and cyclopropyl (3l) aldehydes were smoothly transformed into the corresponding products 7i71 in $61 \%$ to $84 \%$ yields, albeit an ether derived but enolisable aldehyde $\mathbf{3 m}$ led to a less efficient reaction providing the product $\mathbf{7} \mathbf{m}$ with moderate $37 \%$ yield. Importantly, in every case only one diastereoisomer was obtained and isolated in this series. Then, we turned our attention to the use of various pyridazinones $\mathbf{5 b - f}$ in order to probe the diastereoselectivity issue of this MCR. Even with a small methyl group on pyridazinone $\mathbf{5 b}$, the corresponding major diastereoisomers of diazabicyclononanediones $\mathbf{7 n}$ and $\mathbf{7 s}$ were isolated in $47 \%$ and $57 \%$ yields (easily separated by column chromatography) from the crude mixture displaying 
promising dr ranging from 82:18 to 85:15. The diastereoisomeric excesses were markedly improved by means of cyclic hydrazines $\mathbf{5}$ flanked by more hindered substituents to afford ethyl (7o, 55\%, 92:8 dr), allyl (7p, 64\%, 90:10 dr) and esters (7q, 64\%, 85:15 dr and 7r, 87\%, >94:6 dr). Excellent results were also obtained with an aliphatic aldehyde to give the ester-derived diazabicyclononanedione $\mathbf{7 u}$ with 86\% yield and high >95:5 dr although a limitation was encountered with pyridazinone $\mathbf{5 b}$ with a small methyl-group yielding product $7 t$ in $17 \%$ (82:18 dr).

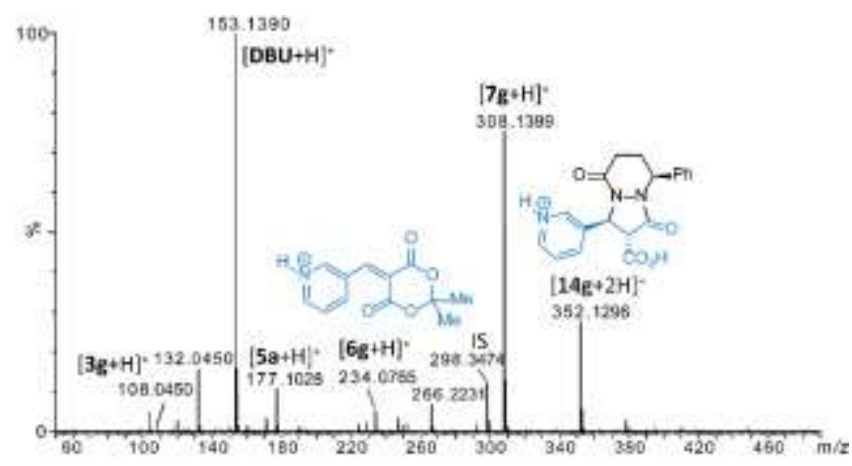

Figure 1. ESI-IMS-MS insight into reaction intermediates (see also Table 2) after 60 minutes. The reaction was carried out at $30{ }^{\circ} \mathrm{C}$ to prevent a too fast process. IS: internal standard.

Getting experimental evidence of intermediates involved into a complex organocatalyzed MCR is not a trivial task, especially with labile Meldrum's acid derivatives. ${ }^{13}$ By means of soft electrospray ionization mass spectrometry (ESI-MS), ${ }^{13}$ key intermediates were sought at various time intervals. We reasoned that the pyridyl moiety onto aldehyde $\mathbf{3 g}$ would afford an ideal probe to detect ions (protonated intermediates) in positive mode. To our delight, the ESI-MS and MS/MS data as well as accurate mass measurements were consistent with the pyridazinone 5a and 3pyridyl-carboxaldehyde $\mathbf{3 g}$ reactants, the DBU catalyst and the product $\mathbf{7 g}$ (Figure 1, see also SI). Importantly, the ions $[6 \mathbf{g}+\mathbf{H}]^{+}$and $[\mathbf{1 4 g}+\mathbf{2 H}]^{+}$of putative intermediates $\mathbf{6 g}$ and $\mathbf{1 4 g}$, the precursor undergoing the decarboxylative-protonation event, were clearly identified as well. Next, the coupling of ion mobility spectrometry (IMS) with MS, allowed the determination of the experimental collision cross section (CCS) values, ${ }^{14}$ known to be chemical descriptors that give information about the shape of ionic compounds in the gas phase, increasing the identification confidence. Accordingly, the experimental ${ }^{\mathrm{T}}{ }_{\mathrm{CCS}} \mathrm{He}$ values match with the calculated ${ }^{\mathrm{TM}} \mathrm{CCS}_{\mathrm{He}}$ (using trajectory method) obtained from the DFT studies, which confirm the expected structures (Table 2). ${ }^{15}$ Then, the key intermediate of the KMC sequence was highlighted and the carboxylate ion $\mathbf{1 4 g}$ (detected as $[14 \mathrm{~g}+2 \mathrm{H}]^{+}$) is, likely, the species (before the resting state) which was detected by ${ }^{1} \mathrm{H}$ NMR when reactions were performed at temperature lower than $50{ }^{\circ} \mathrm{C}$, due to an energy demanding decarboxylation reaction (see SI).

Table 2. Accurate mass measurements and CCS estimation

$\begin{array}{llllll}\text { Ion } & \text { Measured } & \text { Mass } & \text { er- } & { }^{\mathrm{TWCCS}_{\mathrm{He}}}{ }^{a} & { }^{\mathrm{TMCCS}} \mathrm{He}^{b} \\ {[\mathrm{M}+\mathrm{H}]^{+}} & (m / z) & \text { ror } & \left(\AA^{2}\right) & \left(\AA^{2}\right) \\ & & (\mathrm{ppm}) & & \\ \mathbf{D B U} & 153.1389 & 2.6 & 72.8 \pm 0.4 & 71 \pm 1 \\ \mathbf{3 g} & 108.0448 & 3.7 & 63.2 \pm 0.7 & 56 \pm 1 \\ \mathbf{5 a} & 177.1027 & 2.8 & 79.2 \pm 0.3 & 77 \pm 1 \\ \mathbf{7 g} & 308.1398 & 1.3 & 108.3 \pm 0.4 & 109 \pm 1 \\ \mathbf{6 g} & 234.0766 & 2.1 & 93.0 \pm 0.8 & 91 \pm 1 \\ \mathbf{1 4 g} & 352.1297 & 1.4 & 122.3 \pm 0.9 & 120 \pm 1\end{array}$

${ }^{a}$ Experimental CCS. ${ }^{b}$ Calculated CCS. 
Based on the results of the ESI-MS and IMS-MS studies, we proposed the catalytic cycle depicted in Scheme 3. The first step would be a reversible DBU-promoted Knoevenagel condensation between an aldehyde $\mathbf{3}$ and Meldrum's acid 4, yielding the alkylidene Meldrum's acid 6. Next, a base promoted aza-Michael driven by a hydrogenbonding takes place between 5 and $\mathbf{6}$ (via transition state A), ${ }^{16}$ whose substituents of each partner are away to each other to minimize the steric repulsion to give rise to the stereoselective formation of adduct $\mathbf{B}$. Then, a facile cyclocondensation (-acetone) would take place on the more nucleophilic secondary amine of the hydrazine function securing the first chemoselective conjugate addition and affording the ammonium carboxylate 14. Eventually, a likely energy demanding decarboxylation of $\mathbf{1 4}$ can take place, followed by a protonation of the transient enolate, leading to the desired product 7 .

Scheme 3. Proposed catalytic cycle and model of induction

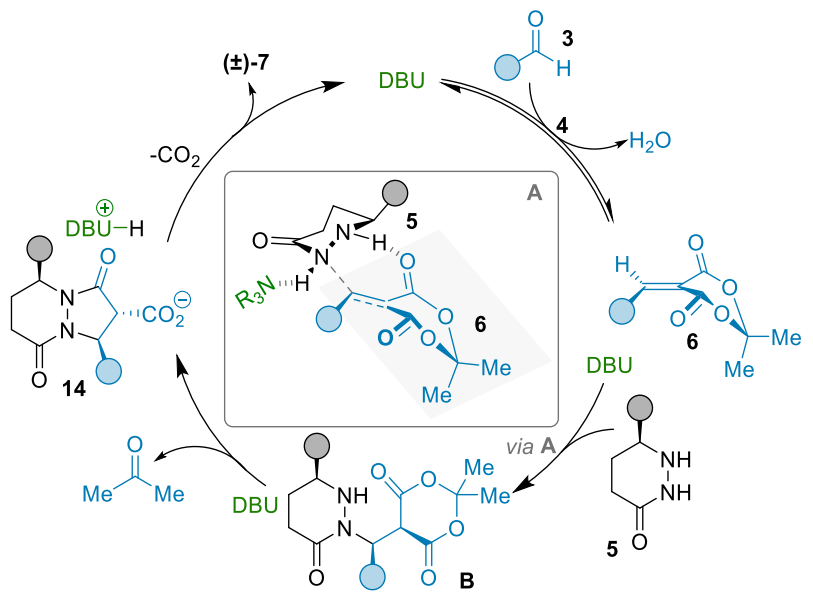

Then, we undertook a preliminary functionalization investigation of these novel heterocylic platforms (Scheme 4).

\section{Scheme 4. Chemical transformations}

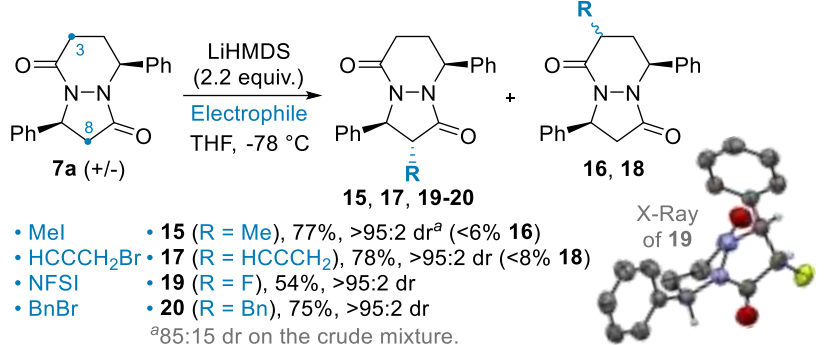

Interestingly, by means of an excess of LiHMDS, the initially formed or more reactive five membered-ring lithium-enolate species of precursor 7a underwent a selective methylation reaction at 8-position with 85:15 $\mathrm{dr}$ (15). The major diastereosiomer 15 was isolated with 77\% yield but with a small amount of the inseparable and likely other regioisomer 16. By means of a more sterically hindered electrophile, the propargylation reaction took place with a high $>95: 5 \mathrm{dr}$ to give the isolated product $\mathbf{1 7}$ and traces of $\mathbf{1 8}$. However, fluorination and benzylation reactions occurred with an excellent regio- and diastereoselectivity (>95:5 dr) to furnish the corresponding products $\mathbf{1 9}$ and $\mathbf{2 0}$ in $54 \%$ and $75 \%$ yields respectively.

\section{CONCLUSION}

In summary, a DBU-based organocatalyzed multicomponent KMC reaction highlights a key chemo-selective aza-Michael reaction of pyridazinones to alkylidene Meldrum's acid intermediates to provide a highly diastereoselective synthesis of original 1,6-diazabicyclo[4.3.0]nonane-2,7-dione derivatives. In line with preliminary promising results, the regio- and stereoselective transformation of these novel small-heterocyclic platforms is currently under investigation to move towards more complex architectures.

\section{EXPERIMENTAL SECTION}

General Information. Reactions were performed using oven dried glassware under inert atmosphere of dry argon 
or nitrogen with freshly distilled or purified aldehydes. Internal NMR standards were distilled prior to use. Grignard reagents and organolithium were titrated using reported procedure prior to use. Unless otherwise noted, all reagentgrade chemicals and solvents were obtained from commercial suppliers and were used as received. THF, PhMe, MeCN and $\mathrm{CH}_{2} \mathrm{Cl}_{2}$ were dried over MBRAUN MB SPS-800 Apparatus. Reactions were monitored by thin-layer chromatography with silica gel $60 \mathrm{~F}_{254}$ pre-coated aluminium plates (0.25 mm). Visualization was performed under UV light, phosphomolybdic acid or $\mathrm{KMnO}_{4}$ oxidation. Chromatographic purification of compounds was achieved with 60 silica gel (40-63 $\mu \mathrm{m})$. Melting points were measured on a WME Köfler hot-stage (Stuart SMP3) and are uncorrected. Infrared spectra (IR) were recorded on a PerkinElmer Spectrum 100 Series FT-IR spectrometer. Liquids and solids were applied onto the Single Reflection Attenuated Total Reflectance (ATR) Accessories. Data are reported in $\mathrm{cm}^{-1} \cdot{ }^{1} \mathrm{H}$ NMR spectra (300 MHz), ${ }^{13} \mathrm{C}\left\{{ }^{1} \mathrm{H}\right\}$ NMR spectra $(75 \mathrm{MHz})$ and ${ }^{19} \mathrm{~F}$ NMR spectra $(282 \mathrm{~Hz})$ were recorded on a Bruker Avance300. Data appear in the following order: chemical shifts in ppm which were referenced to the internal solvent signal, multiplicity $(s$, singlet; $d$, doublet; $t$, triplet; $q$, quadruplet; hept, heptuplet; $m$, multiplet, $A B, \mathrm{AB}$ system; $b r$, broad), coupling constant $J$ in Hertz and number of protons. Accurate Mass measurements (HRMS) were performed by the Mass Spectrometry Laboratory of the University of Rouen using a Waters LCT Premier XE mass spectrometer or a JEOL AccuTOF 4G mass spectrometer. HPLC analyses were performed with Daicel Chiralpak ${ }^{\circledR}$ columns (250 mm x $4.6 \mathrm{~mm}$ ). A Thermo Scientific ${ }^{\mathrm{TM}}$ UltiMate $^{\mathrm{TM}}$ DAD-3000 UV detector was used. X-Ray structures are depicted as thermal ellipsoids at a 50\% probability level using the following colour code: $\mathrm{C}$, grey; $\mathrm{H}$, white; $\mathrm{F}$, light green; $\mathrm{N}$, light purple; $\mathrm{O}$, red. The supplementary crystallographic data can be obtained free of charge from the Cambridge Crystallographic Data Centre via www.ccdc.cam.ac.uk/structures/.

General procedure for the synthesis of tetrahydropyridazinone 5 by hydrazone reduction of dihydropyridazinone. 6Substituted-4,5-dihydropyridazin-3(2H)-one (1 equiv) was dissolved in $\mathrm{MeOH}(0.2 \mathrm{M})$ at RT and the mixture was cooled to $0{ }^{\circ} \mathrm{C} . \mathrm{NaBH}_{3} \mathrm{CN}$ (2 equiv) was added. The $\mathrm{pH}$ of the reaction mixture was maintained between 4 and 5 by dropwise addition of $\mathrm{HCl}\left(37 \% \mathrm{v} / \mathrm{v}\right.$ in $\left.\mathrm{H}_{2} \mathrm{O}\right)$ and the reaction mixture was allowed to reach RT. During the required time, the $\mathrm{pH}$ of the reaction mixture was maintained between 4 and 5 by dropwise addition of $\mathrm{HCl}\left(37 \% \mathrm{v} / \mathrm{v}\right.$ in $\left.\mathrm{H}_{2} \mathrm{O}\right)$. The $\mathrm{pH}$ of the reaction mixture was then adjusted between 8 and 9 by dropwise addition of $\mathrm{NaOH} 4 \mathrm{M}$ at RT. The solution was stirred for $1 \mathrm{~h}$ at RT and concentrated under reduced pressure. The paste was partially dissolved in $\mathrm{MeOH}$, adsorbed on silica and purified by flash column chromatography on silica gel.

6-Phenyltetrahydropyridazin-3(2H)-one (5a). The title compound was prepared according to the above general procedure from 6-phenyl-4,5-dihydropyridazin-3(2H)-one ${ }^{17}(6.1$ g, 34.8 mmol, 1 equiv) and $\mathrm{NaBH}_{3} \mathrm{CN}$ (4.4 g, $69.7 \mathrm{mmol}, 2$ equiv) in $\mathrm{MeOH}(162.0 \mathrm{~mL}, 0.2 \mathrm{M}$ ) at a pH between 3 and 4 in 7 h. Flash column chromatography over silica gel $\left(\mathrm{CH}_{2} \mathrm{Cl}_{2} / \mathrm{MeOH} 95: 5 \mathrm{v} / \mathrm{v}\right.$ to $\left.90: 10 \mathrm{v} / \mathrm{v}\right)$ afforded the title compound as a white powder (2.3 g, 38\%). $R_{f}=0.29$ $\left(\mathrm{CH}_{2} \mathrm{Cl}_{2} / \mathrm{MeOH} 95: 5 \mathrm{v} / \mathrm{v}\right) .{ }^{1} \mathrm{H}$ NMR $\left(300 \mathrm{MHz}, \mathrm{CDCl}_{3}\right) \delta_{\mathrm{H}}$ 
7.40-7.28 (m, 5H), 7.13 (br s, 1H), 4.18 (br s, 1H), 3.99 (br s, 1H), $2.74-2.56(\mathrm{~m}, 2 \mathrm{H}), 2.48-2.38(\mathrm{~m}, 1 \mathrm{H}), 2.27-2.14(\mathrm{~m}$, 1H). ${ }^{13} \mathrm{C}\left\{{ }^{1} \mathrm{H}\right\}$ NMR (75 MHz, $\left.\mathrm{CDCl}_{3}\right) \delta_{\mathrm{C}} 171.8(\mathrm{C}), 139.6(\mathrm{C})$, $128.8(\mathrm{CH}), 128.0(\mathrm{CH}), 126.8(\mathrm{CH}), 57.8(\mathrm{CH}), 29.2\left(\mathrm{CH}_{2}\right)$, $29.0\left(\mathrm{CH}_{2}\right)$. HRMS (API/TOF): $m / z:[\mathrm{M}+\mathrm{H}]^{+}$Calcd for $\mathrm{C}_{10} \mathrm{H}_{13} \mathrm{~N}_{2} \mathrm{O}$ 177.1023; Found 177.1030. The spectroscopic data are in agreement with literature. ${ }^{17}$ The enantiomers were separated by semi-preparative HPLC $(\mathrm{OJ}-\mathrm{H}, \mathrm{PrOH} / n$ heptane $30 / 70$, flow rate $=15.0 \mathrm{~mL} / \mathrm{min}, l=213 \mathrm{~nm}) t_{\mathrm{R}}=$ $11.8 \mathrm{~min}$ (first),14.0 $\mathrm{min}$ (second). Both enantiomers were recovered in 99\% ee. Remark: It was observed sometimes that 5a was partially oxidize into 6-phenyl-4,5-dihydropyridazin-3(2H)-one during storage at RT.

6-Methyltetrahydropyridazin-3(2H)-one (5b). The title compound was prepared according to the above general procedure from 6-methyl-4,5-dihydropyridazin-3(2H)one ${ }^{18}$ (474.8 mg, 4.2 mmol, 1 equiv) and $\mathrm{NaBH}_{3} \mathrm{CN}$ (532.0 mg, 8.5 mmol, 2 equiv) in $\mathrm{MeOH}$ (19.7 mL, $0.2 \mathrm{M}$ ) in $6 \mathrm{~h}$. Flash column chromatography over silica gel $\left(\mathrm{CH}_{2} \mathrm{Cl}_{2} / \mathrm{MeOH} 94: 6\right.$ $\mathrm{v} / \mathrm{v}$ ) afforded the title compound as a white powder (299.4 mg, 82\%). $R_{f}=0.17\left(\mathrm{CH}_{2} \mathrm{Cl}_{2} / \mathrm{MeOH} 94: 6 \mathrm{v} / \mathrm{v}\right) \cdot{ }^{1} \mathrm{H}$ NMR $(300$ $\left.\mathrm{MHz}, \mathrm{CDCl}_{3}\right) \delta_{\mathrm{H}} 7.10(\mathrm{br} \mathrm{s}, 1 \mathrm{H}), 3.55(\mathrm{br} \mathrm{s}, 1 \mathrm{H}), 3.14(\mathrm{dtd}, J=$ 9.4, 6.6, 4.2 Hz, 1H), 2.58-2.40 (m, 2H), 2.08 (dddd, $J=13.8$, 7.1, 4.9, 4.2 Hz, 1H), 1.59 (dtd, $J=13.8,9.4,8.2 \mathrm{~Hz}, 1 \mathrm{H}), 1.15$ (d, $J=6.6 \mathrm{~Hz}, 3 \mathrm{H})$. HRMS (ESI/TOF) $m / z:[\mathrm{M}+\mathrm{H}]^{+}$Calcd for $\mathrm{C}_{5} \mathrm{H}_{11} \mathrm{~N}_{2} \mathrm{O}$ 115.0866; Found 115.0863. The spectroscopic data are in agreement with literature. ${ }^{19}$

Methyl 6-oxohexahydropyridazine-3-carboxylate (5e). The title compound was prepared according to the above general procedure from methyl 6-oxo-4,5-dihydropyridazine3-carboxylate ${ }^{20}$ (760 mg, $4.87 \mathrm{mmol}, 1$ equiv) and $\mathrm{NaBH}_{3} \mathrm{CN}$
(612.1 mg, $9.7 \mathrm{mmol}, 2$ equiv) in $\mathrm{MeOH}$ (24.4 mL, 0.2 $\mathrm{M}$ ) in 3 h. Flash column chromatography over silica gel $\left(\mathrm{CH}_{2} \mathrm{Cl}_{2} / \mathrm{MeOH} 94: 6 \mathrm{v} / \mathrm{v}\right)$ afforded the title compound as a white powder (399 mg, 52\%). m.p. $96-97^{\circ} \mathrm{C}$. IR (neat) $v_{\max }$ 3261, 2963, 1675, 1401, $1222 \mathrm{~cm}^{-1} .{ }^{1} \mathrm{H}$ NMR (300 MHz, $\left.\mathrm{CDCl}_{3}\right) \delta_{\mathrm{H}} 7.81($ br s, $1 \mathrm{H}), 4.42($ br s, $1 \mathrm{H}), 3.80(\mathrm{dd}, J=8.5$, $6.2 \mathrm{~Hz}, 1 \mathrm{H}), 3.74(\mathrm{~s}, 3 \mathrm{H}), 2.57-2.40(\mathrm{~m}, 2 \mathrm{H}), 2.33-2.22(\mathrm{~m}$, 1H), $2.12-2.00(\mathrm{~m}, 1 \mathrm{H}) .{ }^{13} \mathrm{C}\left\{{ }^{1} \mathrm{H}\right\} \operatorname{NMR}\left(75 \mathrm{MHz}^{\mathrm{CDCl}}{ }_{3}\right) \delta_{\mathrm{C}}$ 172.6, 171.9, 56.0, 52.5, 28.5, 26.4. HRMS (API/TOF) $m / z$ : $[\mathrm{M}+\mathrm{MeCN}+\mathrm{H}]^{+}$Calcd for $\mathrm{C}_{8} \mathrm{H}_{14} \mathrm{~N}_{3} \mathrm{O}_{3} \quad 200.1029 ;$ Found 200.1031.

tert-Butyl 6-oxohexahydropyridazine-3-carboxylate (5f). The title compound was prepared according to the above general procedure from tert-butyl 6-oxo-4,5-dihydropyridazine-3-carboxylate ${ }^{21}(650 \mathrm{mg}, 3.28 \mathrm{mmol}, 1$ equiv) and $\mathrm{NaBH}_{3} \mathrm{CN}$ (412.2 mg, $6.6 \mathrm{mmol}, 2$ equiv) in $\mathrm{MeOH}$ (16.4 $\mathrm{mL}, 0.2 \mathrm{M}$ ) in $4 \mathrm{~h}$. Flash column chromatography over silica gel $\left(\mathrm{CH}_{2} \mathrm{Cl}_{2} / \mathrm{MeOH} 97: 3 \mathrm{v} / \mathrm{v}\right)$ afforded the title compound as a white powder (353 mg, 54\%). m.p. $109-110^{\circ} \mathrm{C}$. IR (neat) $\nu_{\max } 3247,2977,2927,1723,1645,1364,1235,1155,915$, $736 \mathrm{~cm}^{-1} .{ }^{1} \mathrm{H}$ NMR $\left(300 \mathrm{MHz}, \mathrm{CDCl}_{3}\right) \delta_{\mathrm{H}} 7.32(\mathrm{br} \mathrm{s}, 1 \mathrm{H}), 4.13$ (br s, $1 \mathrm{H}), 3.68(\mathrm{dd}, J=8.7,6.0 \mathrm{~Hz}, 1 \mathrm{H}), 2.58-2.41(\mathrm{~m}, 2 \mathrm{H})$, 2.32-2.21 (m, 1H), 2.09-1.97 (m, 1H), $1.46(\mathrm{~s}, 9 \mathrm{H}) .{ }^{13} \mathrm{C}\left\{{ }^{1} \mathrm{H}\right\}$ NMR (75 MHz, $\left.\mathrm{CDCl}_{3}\right) \delta_{\mathrm{C}} 172.4,170.7,82.4,56.6,28.6,28.0$ (3C), 26.8. HRMS (API/TOF) $m / z:[\mathrm{M}+\mathrm{H}]^{+}$Calcd for $\mathrm{C}_{9} \mathrm{H}_{17} \mathrm{~N}_{2} \mathrm{O}_{3}$ 201.1234; Found 201.1237.

\section{General procedure for the synthesis of tetrahydropyridazi-} nones $\mathbf{5}$ via nucleophilic addition on dihydropyridazinone. 4,5-Dihydropyridazin-3(2H)-one ${ }^{12}$ (1 equiv) was introduced in dried glassware under Ar atmosphere. Three purge-and-refill procedures were carried out (subjected to 
vacuum and then refilled with argon), then anhydrous toluene $(0.1 \mathrm{M})$ was added. The mixture was stirred at $0{ }^{\circ} \mathrm{C}$, then appropriate Grignard reagent (4 equiv) or organolithium (2.2 equiv) was added dropwise. The ice bath was removed after $10 \mathrm{~min}$. After $3.5 \mathrm{~h}$, the reaction was quenched at $0{ }^{\circ} \mathrm{C}$ by aqueous saturated $\mathrm{NH}_{4} \mathrm{Cl}$ for $30 \mathrm{~min}$. The reaction mixture was extracted with EtOAc (3 times). The combined organic layers were dried over $\mathrm{Na}_{2} \mathrm{SO}_{4}$, filtered and concentrated under reduced pressure. The crude product was then purified by flash column chromatography over silica gel.

6-Phenyltetrahydropyridazin-3(2H)-one (5a). The title compound was prepared according to the above general procedure from 4,5-dihydropyridazin-3(2H)-one (4.9 g, 50.0 mmol, 1 equiv) and PhLi (53.7 mL, $110.0 \mathrm{mmol}, 2.2$ equiv, $2.05 \mathrm{M}$ in $\left.n \mathrm{Bu}_{2} \mathrm{O}\right)$ in anhydrous toluene (500 mL, $\left.0.1 \mathrm{M}\right)$. Flash column chromatography over silica gel $\left(\mathrm{CH}_{2} \mathrm{Cl}_{2} / \mathrm{MeOH} 95: 5 \mathrm{v} / \mathrm{v}\right.$ to $\left.90: 10 \mathrm{v} / \mathrm{v}\right)$ afforded the title compound as a white powder (3.4 g, 39\%). $R_{f}=0.29$ $\left(\mathrm{CH}_{2} \mathrm{Cl}_{2} / \mathrm{MeOH} 95: 5 \mathrm{v} / \mathrm{v}\right)$. The spectroscopic data are in agreement with literature. ${ }^{17}$

6-Ethyltetrahydropyridazin-3(2H)-one (5c). The title compound was prepared according to the above general procedure from 4,5-dihydropyridazin-3(2H)-one (14.7 mg, 0.15 mmol, 1 equiv) and EtMgBr (140.8 $\mu \mathrm{L}, 0.6 \mathrm{mmol}, 4$ equiv, 4.3 $\mathrm{M}$ in $\left.\mathrm{Et}_{2} \mathrm{O}\right)$ in anhydrous toluene $(1.5 \mathrm{~mL}, 0.1 \mathrm{M}) .$. Flash column chromatography over silica gel $\left(\mathrm{CH}_{2} \mathrm{Cl}_{2} / \mathrm{MeOH}\right.$ 97:3 v/v to $96: 4 \mathrm{v} / \mathrm{v}$ ) afforded the title compound as a yellow oil (6.5 mg, 34\%). $R_{f}=0.16\left(\mathrm{CH}_{2} \mathrm{Cl}_{2} / \mathrm{MeOH} 97: 3 \mathrm{v} / \mathrm{v}\right)$. IR (neat) $v_{\max } 3227,2964,2934,1639,1400 \mathrm{~cm}^{-1} .{ }^{1} \mathrm{H}$ NMR $(300 \mathrm{MHz}$, $\left.\mathrm{CDCl}_{3}\right) \delta_{\mathrm{H}} 7.08(\mathrm{br} \mathrm{s}, 1 \mathrm{H}), 3.61$ (br s, 1H), 2.93-2.84 (m, 1H), 2.57-2.39 (m, 2H), 2.15-2.05 (m, 1H), 1.64-1.34 (m, 3H), $0.98(\mathrm{t}, J=7.4 \mathrm{~Hz}, 3 \mathrm{H}) \cdot{ }^{13} \mathrm{C}\left\{{ }^{1} \mathrm{H}\right\} \mathrm{NMR}\left(75 \mathrm{MHz}, \mathrm{CDCl}_{3}\right) \delta_{\mathrm{c}} 172.8$ (C), $56.5(\mathrm{CH}), 29.0\left(\mathrm{CH}_{2}\right), 28.9\left(\mathrm{CH}_{2}\right), 26.6\left(\mathrm{CH}_{2}\right), 10.9\left(\mathrm{CH}_{3}\right)$. HRMS (ESI/TOF) $m / z$ : [M+H] ${ }^{+}$Calcd for $\mathrm{C}_{6} \mathrm{H}_{13} \mathrm{~N}_{2} \mathrm{O}$ 129.1023; Found 129.1027.

6-Allyltetrahydropyridazin-3(2H)-one (5d). The title compound was prepared according to the above general procedure from 4,5-dihydropyridazin-3(2H)-one (147.2 mg, 1.5 mmol) and allylMgBr (6.0 mL, $6 \mathrm{mmol}, 4$ equiv, $1.0 \mathrm{M}$ in $\left.\mathrm{Et}_{2} \mathrm{O}\right)$ in anhydrous toluene $(15.0 \mathrm{~mL}, 0.1 \mathrm{M}) .$. Flash column chromatography over silica gel $\left(\mathrm{CH}_{2} \mathrm{Cl}_{2} / \mathrm{MeOH} 95: 5 \mathrm{v} / \mathrm{v}\right)$ afforded the title compound as a yellow oil (51.7 mg, 25\%). $R_{f}$ $=0.15\left(\mathrm{CH}_{2} \mathrm{Cl}_{2} / \mathrm{MeOH} 95: 5 \mathrm{v} / \mathrm{v}\right)$. IR (neat) $v_{\max } 3221,2930$, $1638,911,440 \mathrm{~cm}^{-1} .{ }^{1} \mathrm{H}$ NMR $\left(300 \mathrm{MHz}^{\mathrm{CDCl}} 3\right) \delta_{\mathrm{H}} 6.99(\mathrm{br}$ s, $1 \mathrm{H}), 5.88-5.74(\mathrm{~m}, 1 \mathrm{H}), 5.16-5.12(\mathrm{~m}, 1 \mathrm{H}), 5.09(\mathrm{t}, J=1.3$ $\mathrm{Hz}, 1 \mathrm{H}), 3.70$ (brs, 1H), 3.14-3.05 (m, 1H), 2.58-2.40 (m, 2H), 2.33-2.19 (m, 2H), 2.17-2.05 (m, 1H), 1.73-1.59 (m, 1H). ${ }^{13} \mathrm{C}\left\{{ }^{1} \mathrm{H}\right\}$ NMR (75 MHz, $\left.\mathrm{CDCl}_{3}\right) \delta_{\mathrm{C}} 172.5(\mathrm{C}), 134.2(\mathrm{CH})$, $117.9\left(\mathrm{CH}_{2}\right), 54.4(\mathrm{CH}), 37.8\left(\mathrm{CH}_{2}\right), 28.8\left(\mathrm{CH}_{2}\right), 28.5\left(\mathrm{CH}_{2}\right)$. HRMS (ESI/TOF) $m / z:[\mathrm{M}+\mathrm{H}]^{+}$Calcd for $\mathrm{C}_{7} \mathrm{H}_{13} \mathrm{~N}_{2} \mathrm{O}$ 141.1023; Found 141.1022.

General procedure for the diastereoselective preparation of 1,6-diazabicyclo[4.3.0]nonane-2,7-diones 7. To a mixture of tetrahydropyridazin-3(2H)-one 5 ( $0.3 \mathrm{mmol}, 1$ equiv) and Meldrum's acid 4 (56.2 mg, $0.4 \mathrm{mmol}, 1.3$ equiv) under Ar, THF (3 mL, $0.1 \mathrm{M})$ and DBU $(4.5 \mu \mathrm{L}, 0.03 \mathrm{mmol}, 0.01$ equiv) were added. Aldehyde 3 (0.3 mmol, 1 equiv) was then introduced at room temperature. The tube was sealed, and the mixture was stirred at $50^{\circ} \mathrm{C}$ (oil bath temperature) during the required time. Then the reaction mixture was allowed to reach room temperature, diluted by $\mathrm{CH}_{2} \mathrm{Cl}_{2}$ and washed with a solution of $\mathrm{Na}_{2} \mathrm{CO}_{3}\left(10 \% \mathrm{w} / \mathrm{w}\right.$ in $\left.\mathrm{H}_{2} \mathrm{O}, 10 \mathrm{~mL}\right)$. 
The aqueous layer was then extracted twice by $\mathrm{CH}_{2} \mathrm{Cl}_{2}(2 \mathrm{x}$ $15 \mathrm{~mL}$ ). The combined organic layers were dried over $\mathrm{Na}_{2} \mathrm{SO}_{4}$, filtered and concentrated under reduced pressure. The crude product was then purified by flash column chromatography over silica gel. Unless otherwise noted, the crude reaction mixture showed a diastereoisomeric ratio > 95:5 by means of ${ }^{1} \mathrm{H}$ NMR.

5,9-Diphenyl-1,6-diazabicyclo[4.3.0]nonane-2,7-dione (7a). The title compound was prepared according to the above general procedure from $5 \mathbf{5}(52.9 \mathrm{mg}, 0.3 \mathrm{mmol}, 1$ equiv) and benzaldehyde 3a (30.5 $\mu \mathrm{L}, 0.3 \mathrm{mmol}, 1$ equiv) in 16 hours. Flash column chromatography over silica gel (PE/EtOAc $10: 90 \mathrm{v} / \mathrm{v}$ to $\mathrm{EtOAc} / \mathrm{MeOH} 98: 2 \mathrm{v} / \mathrm{v}$ ) afforded the title compound as a whitish oil (74.6 mg, 81\%, >95:5 dr). On $1 \mathrm{mmol}$ scale the product was obtained from $\mathbf{5 a}(176.2 \mathrm{mg}, 1.0$ mmol, 1.0 equiv), Meldrum's acid 4 (187.4 mg, 1.3 mmol, 1.3 equiv) and benzaldehyde $3 a(101.7 \mu \mathrm{L}, 1.0 \mathrm{mmol}, 1.0$ equiv) with DBU (15.0 $\mu \mathrm{L}, 0.1 \mathrm{mmol}, 0.01$ equiv) and THF (10 mL, $0.1 \mathrm{M}$ ) in $73 \%$ yield $(222.8 \mathrm{mg}, 73 \%,>95: 5 d r)$. Remark: a small-scale reaction with enantiopure 5 a $(17.0 \mathrm{mg}, 0.1$ mmol, 1 equiv, 99\% ee) afforded the enantiopure compound 7a as a whitish oil (11.8 mg, 40\%, >95:5 dr, 99\% ee). HPLC (IE, $i \operatorname{PrOH} / n$-heptane $=50 / 50$, flow rate $=1.0 \mathrm{~mL} / \mathrm{min}, l=$ $204 \mathrm{~nm}) t_{\mathrm{R}}=13.8$ (major), 15.6 (minor). $R_{f}=0.22$ (EtOAc). IR (neat) $v_{\max } 3037,2931,1699,1647,1348,1331,1254$, 909, 696, $504 \mathrm{~cm}^{-1} .{ }^{1} \mathrm{H}$ NMR $\left(300 \mathrm{MHz}, \mathrm{CDCl}_{3}\right) \delta_{\mathrm{H}} 7.41-7.33$ (m, 5H), 7.31-7.26 (m, 3H), 7.18-7.13 (m, 2H), $5.86(\mathrm{dd}, J=$ 10.5, 2.7 Hz, 1H), 5.59 (t, $J=5.0 \mathrm{~Hz}, 1 \mathrm{H}), 3.31$ (dd, $J=17.4$, $10.5 \mathrm{~Hz}, 1 \mathrm{H}), 2.91(\mathrm{dd}, J=17.4,2.7 \mathrm{~Hz}, 1 \mathrm{H}), 2.53-2.47(\mathrm{~m}$, 2H), 2.36-2.29 (m, 2H). ${ }^{13} \mathrm{C}\left\{{ }^{1} \mathrm{H}\right\}$ NMR $\left(75 \mathrm{MHz}, \mathrm{CDCl}_{3}\right) \delta_{\mathrm{c}}$ 165.7 (C), 165.1 (C), 139.0 (C), 137.9 (C), 129.0 (CH), 128.9
(CH), $128.5(\mathrm{CH}), 128.1(\mathrm{CH}), 126.5(\mathrm{CH}), 126.0(\mathrm{CH}), 55.1$ (CH), $52.3(\mathrm{CH}), 37.3\left(\mathrm{CH}_{2}\right), 28.0\left(\mathrm{CH}_{2}\right), 26.1\left(\mathrm{CH}_{2}\right) . \mathrm{HRMS}$ (ESI/TOF) $m / z:[\mathrm{M}+\mathrm{H}]^{+}$Calcd for $\mathrm{C}_{19} \mathrm{H}_{19} \mathrm{~N}_{2} \mathrm{O}_{2}$ 307.1441; Found 307.1444.

\section{9-(2-Methylphenyl)-5-phenyl-1,6-diazabicyclo[4.3.0]non-}

ane-2,7-dione (7b). The title compound was prepared according to the above general procedure from $\mathbf{5 a}(52.9 \mathrm{mg}$, $0.3 \mathrm{mmol}, 1$ equiv) and 2-methylbenzaldehyde $\mathbf{3 b}(34.9 \mu \mathrm{L}$, $0.3 \mathrm{mmol}, 1$ equiv) in 16 hours. Flash column chromatography over silica gel (PE/EtOAc 10:90 v/v to EtOAc/MeOH $98: 2 \mathrm{v} / \mathrm{v}$ ) afforded the title compound as a colourless oil (54.7 mg, 57\%, >95:5 dr). $R_{f}=0.30(\mathrm{EtOAc} / \mathrm{MeOH} \mathrm{98:2} \mathrm{v/v).}$ IR (neat) $v_{\max } 2924,1694,1649,1348,1328,1252,911,725$, $699,495,462 \mathrm{~cm}^{-1} .{ }^{1} \mathrm{H}$ NMR $\left(300 \mathrm{MHz}, \mathrm{CDCl}_{3}\right) \delta_{\mathrm{H}} 7.40-7.30$ (m, 5H), 7.21-7.19 (m, 2H), 7.13-7.07 (m, 2H), $5.91(\mathrm{dd}, J=$ 10.7, $3.5 \mathrm{~Hz}, 1 \mathrm{H}), 5.62(\mathrm{t}, J=5.2 \mathrm{~Hz}, 1 \mathrm{H}), 3.36(\mathrm{dd}, J=17.3$, $10.7 \mathrm{~Hz}, 1 \mathrm{H}), 2.68(\mathrm{dd}, J=17.3,3.5 \mathrm{~Hz}, 1 \mathrm{H}), 2.71-2.29(\mathrm{~m}$, 4H), $2.45(\mathrm{~s}, 3 \mathrm{H}) \cdot{ }^{13} \mathrm{C}\left\{{ }^{1} \mathrm{H}\right\} \mathrm{NMR}\left(75 \mathrm{MHz}, \mathrm{CDCl}_{3}\right) \delta_{\mathrm{c}} 165.73$ (C), 165.67 (C), 137.9 (C), 137.8 (C), 134.9 (C), $131.2(\mathrm{CH})$, $129.0(\mathrm{CH}), 128.3(\mathrm{CH}), 128.2(\mathrm{CH}), 126.5(\mathrm{CH}), 126.3(\mathrm{CH})$, $124.4(\mathrm{CH}), 52.8(\mathrm{CH}), 52.7(\mathrm{CH}), 38.1\left(\mathrm{CH}_{2}\right), 28.4\left(\mathrm{CH}_{2}\right), 25.6$ $\left(\mathrm{CH}_{2}\right), 19.5\left(\mathrm{CH}_{3}\right)$. HRMS (CI/TOF) $\mathrm{m} / \mathrm{z}:[\mathrm{M}+\mathrm{H}]^{+}$Calcd for $\mathrm{C}_{20} \mathrm{H}_{21} \mathrm{~N}_{2} \mathrm{O}_{2}$ 321.1598; Found 321.1589.

\section{9-(3-Methylphenyl)-5-phenyl-1,6-diazabicyclo[4.3.0]non-}

ane-2,7-dione (7c). The title compound was prepared according to the above general procedure from $\mathbf{5 a}(52.9 \mathrm{mg}$, $0.3 \mathrm{mmol}, 1$ equiv) and 3-methylbenzaldehyde 3 c (35.3 $\mu \mathrm{L}$, $0.3 \mathrm{mmol}, 1$ equiv) in 16 hours. Flash column chromatography over silica gel (PE/EtOAc 10:90 v/v to EtOAc/MeOH 98:2 v/v) afforded the title compound as a yellow solid (61.5 mg, 64\%, >95:5 dr). $R_{f}=0.27(\mathrm{EtOAc} / \mathrm{MeOH} \mathrm{98:2} \mathrm{v/v).}$ 
m.p. $87-89{ }^{\circ} \mathrm{C} . \mathrm{IR}$ (neat) $\nu_{\max } 2937,1672,1326,1238,904$, $759,700,623,533,488 \mathrm{~cm}^{-1} .{ }^{1} \mathrm{H}$ NMR $\left(300 \mathrm{MHz}, \mathrm{CDCl}_{3}\right) \delta_{\mathrm{H}}$ 7.29-7.13 (m, 9H), $5.81(\mathrm{dd}, J=10.5,2.7 \mathrm{~Hz}, 1 \mathrm{H}), 5.60(\mathrm{t}, J=$ $5.0 \mathrm{~Hz}, 1 \mathrm{H}), 3.30(\mathrm{dd}, J=17.4,10.5 \mathrm{~Hz}, 1 \mathrm{H}), 2.88$ (dd, $J=17.4$, $2.7 \mathrm{~Hz}, 1 \mathrm{H}), 2.54-2.49(\mathrm{~m}, 2 \mathrm{H}), 2.36-2.28(\mathrm{~m}, 2 \mathrm{H}), 2.32(\mathrm{~s}$, 3H). ${ }^{13} \mathrm{C}\left\{{ }^{1} \mathrm{H}\right\}$ NMR (75 MHz, $\left.\mathrm{CDCl}_{3}\right) \delta_{\mathrm{C}} 165.7(\mathrm{C}), 165.0(\mathrm{C})$, $139.0(\mathrm{C}), 138.8(\mathrm{C}), 137.9(\mathrm{C}), 129.2(\mathrm{CH}), 128.88(\mathrm{CH})$, $128.85(\mathrm{CH}), 128.1(\mathrm{CH}), 127.0(\mathrm{CH}), 126.1(\mathrm{CH}), 123.6(\mathrm{CH})$, $55.1(\mathrm{CH}), 52.2(\mathrm{CH}), 37.5\left(\mathrm{CH}_{2}\right), 28.0\left(\mathrm{CH}_{2}\right), 25.9\left(\mathrm{CH}_{2}\right), 21.5$ $\left(\mathrm{CH}_{3}\right)$. HRMS (CI/TOF) $m / z$ : $[\mathrm{M}+\mathrm{H}]^{+}$Calcd for $\mathrm{C}_{20} \mathrm{H}_{21} \mathrm{~N}_{2} \mathrm{O}_{2}$ 321.1598; Found 321.1604.

\section{9-(4-Methoxyphenyl)-5-phenyl-1,6-diazabicyclo[4.3.0]non-}

ane-2,7-dione $(\mathbf{7 d})$. The title compound was prepared according to the above general procedure from $\mathbf{5 a}(52.9 \mathrm{mg}$, 0.3 mmol, 1 equiv) and $p$-anisaldehyde $3 \mathbf{d}(36.5 \mu \mathrm{L}, 0.3$ mmol, 1 equiv) in 16 hours. Flash column chromatography over silica gel (PE/EtOAc 10:90 v/v to EtOAc/MeOH 98:2 $\mathrm{v} / \mathrm{v}$ ) afforded the title compound as an orange solid (64.4 mg, 64\%, >95:5 dr). $R_{f}=0.19$ (EtOAc). m.p. $75-76{ }^{\circ} \mathrm{C} . \mathrm{IR}$ (neat) $v_{\max } 2928,1670,1513,1329,1251,1174,829,701$, $523 \mathrm{~cm}^{-1} .{ }^{1} \mathrm{H}$ NMR $\left(300 \mathrm{MHz}, \mathrm{CDCl}_{3}\right) \delta_{\mathrm{H}} 7.34-7.23(\mathrm{~m}, 5 \mathrm{H})$, 7.16-7.11 (m, 2H), 6.92-6.87 (m, 2H), $5.82(\mathrm{dd}, J=10.4,2.6$ $\mathrm{Hz}, 1 \mathrm{H}), 5.58(\mathrm{t}, J=4.8 \mathrm{~Hz}, 1 \mathrm{H}), 3.82(\mathrm{~s}, 3 \mathrm{H}), 3.28(\mathrm{dd}, J=17.4$, $10.4 \mathrm{~Hz}, 1 \mathrm{H}), 2.89$ (dd, $J=17.4,2.6 \mathrm{~Hz}, 1 \mathrm{H}), 2.54-2.43(\mathrm{~m}$, 2H), 2.39-2.26 (m, 2H). ${ }^{13} \mathrm{C}\left\{{ }^{1} \mathrm{H}\right\}$ NMR $\left(75 \mathrm{MHz}, \mathrm{CDCl}_{3}\right) \delta_{\mathrm{C}}$ 165.7 (C), 164.6 (C), 159.7 (C), 138.0 (C), 131.1 (C), 128.9 (CH), $128.02(\mathrm{CH}), 128.00(\mathrm{CH}), 125.9(\mathrm{CH}), 114.3(\mathrm{CH}), 55.4$ $(\mathrm{CH}), 54.6(\mathrm{CH}), 52.2\left(\mathrm{CH}_{3}\right), 37.1\left(\mathrm{CH}_{2}\right), 27.9\left(\mathrm{CH}_{2}\right), 26.1$ $\left(\mathrm{CH}_{2}\right)$. HRMS (CI/TOF) $\mathrm{m} / z$ : $[\mathrm{M}+\mathrm{H}]^{+}$Calcd for $\mathrm{C}_{20} \mathrm{H}_{21} \mathrm{~N}_{2} \mathrm{O}_{3}$ 337.1547; Found 337.1564.
9-(4-Chlorophenyl)-5-phenyl-1,6-diazabicyclo[4.3.0]non-

ane-2,7-dione (7e). The title compound was prepared according to the above general procedure from $\mathbf{5 a}(52.9 \mathrm{mg}$, $0.3 \mathrm{mmol}, 1$ equiv) and 4-chlorobenzaldehyde $\mathbf{3 e}(42.2 \mathrm{mg}$, $0.3 \mathrm{mmol}, 1$ equiv) in 16 hours. Flash column chromatography over silica gel (PE/EtOAc $10: 90$ v/v to EtOAc/MeOH $98: 2 \mathrm{v} / \mathrm{v})$ afforded the title compound as a white solid (67.5 $\mathrm{mg}, 66 \%,>95: 5 d r) . R_{f}=0.21(\mathrm{EtOAc} / \mathrm{MeOH} \mathrm{98:2} \mathrm{v/v).} \mathrm{m.p.}$ $137-138{ }^{\circ} \mathrm{C}$. IR (neat) $v_{\max } 2935,1694,1652,1330,1242$, $1174,816,704,525,434 \mathrm{~cm}^{-1} \cdot{ }^{1} \mathrm{H}$ NMR $\left(300 \mathrm{MHz}, \mathrm{CDCl}_{3}\right) \delta_{\mathrm{H}}$ 7.35-7.28 (m, 7H), 7.17-7.14 (m, 2H), $5.81(\mathrm{dd}, J=10.5,2.8$ $\mathrm{Hz}, 1 \mathrm{H}), 5.59(\mathrm{t}, J=5.0 \mathrm{~Hz}, 1 \mathrm{H}), 3.31(\mathrm{dd}, J=17.4,10.5 \mathrm{~Hz}$, 1H), $2.85(\mathrm{dd}, J=17.4,2.8 \mathrm{~Hz}, 1 \mathrm{H}), 2.54-2.49(\mathrm{~m}, 2 \mathrm{H}), 2.37-$ $2.29(\mathrm{~m}, 2 \mathrm{H}) \cdot{ }^{13} \mathrm{C}\left\{{ }^{1} \mathrm{H}\right\}$ NMR (75 MHz, $\left.\mathrm{CDCl}_{3}\right) \delta_{\mathrm{C}} 165.3(\mathrm{C})$, 165.1 (C), 137.7 (C), 137.5 (C), 134.3 (C), $129.1(\mathrm{CH}), 128.9$ (CH), $128.2(\mathrm{CH}), 127.9(\mathrm{CH}), 125.9(\mathrm{CH}), 54.4(\mathrm{CH}), 52.3$ $(\mathrm{CH}), 37.1\left(\mathrm{CH}_{2}\right), 28.0\left(\mathrm{CH}_{2}\right), 25.8\left(\mathrm{CH}_{2}\right)$. HRMS (CI/TOF) $m / z:[\mathrm{M}+\mathrm{H}]^{+}$Calcd for $\mathrm{C}_{19} \mathrm{H}_{18} \mathrm{ClN}_{2} \mathrm{O}_{2}$ 341.1052; Found 341,1059

9-(Naphthalen-2-yl)-5-phenyl-1,6-diazabicyclo[4.3.0]non-

ane-2,7-dione (7f). The title compound was prepared according to the above general procedure from $\mathbf{5 a}(52.9 \mathrm{mg}, 0.3$ mmol, 1 equiv) and 2-naphthaldehyde $3 f(46.9 \mathrm{mg}, 0.3$ mmol, 1 equiv) in 16 hours. Flash column chromatography over silica gel (PE/EtOAc 10:90 v/v to EtOAc/MeOH 98:2 $\mathrm{v} / \mathrm{v}$ ) afforded the title compound as a white powder (76.3 mg, 71\%, >95:5 dr). $R_{f}=0.31$ (EtOAc). m.p. $134-135^{\circ} \mathrm{C} . \mathrm{IR}$ (neat) $v_{\max } 2968,1690,1641,1344,1241,818,755,744$, 704, 531, $479 \mathrm{~cm}^{-1} .{ }^{1} \mathrm{H}$ NMR $\left(300 \mathrm{MHz}, \mathrm{CDCl}_{3}\right) \delta_{\mathrm{H}} 7.88-7.83$ (m, 2H), 7.76-7.71 (m, 2H), 7.53-7.40 (m, 3H), 7.29-7.19 (m, 5H), $6.02(\mathrm{dd}, J=10.6,2.7 \mathrm{~Hz}, 1 \mathrm{H}), 5.63(\mathrm{br} \mathrm{t}, J=5.0 \mathrm{~Hz}$, 
1H), 3.39 (dd, $J=17.4,10.6 \mathrm{~Hz}, 1 \mathrm{H}$ ), 2.99 (dd, $J=17.4,2.7$ $\mathrm{Hz}, 1 \mathrm{H}), 2.57-2.53(\mathrm{~m}, 2 \mathrm{H}), 2.39-2.33(\mathrm{~m}, 2 \mathrm{H}) .{ }^{13} \mathrm{C}\left\{{ }^{1} \mathrm{H}\right\} \mathrm{NMR}$ (75 MHz, $\mathrm{CDCl}_{3}$ ) $\delta_{\mathrm{C}} 165.7$ (C), $165.3(\mathrm{C}), 137.9$ (C), $136.3(\mathrm{C})$, $133.2(\mathrm{C}), 129.2(\mathrm{CH}), 129.0(\mathrm{CH}), 128.22(\mathrm{CH}), 128.20(\mathrm{CH})$, $127.8(\mathrm{CH}), 126.7(\mathrm{CH}), 126.6(\mathrm{CH}), 126.1(\mathrm{CH}), 125.4(\mathrm{CH})$, 124.4 (CH), $55.3(\mathrm{CH}), 52.4(\mathrm{CH}), 37.5\left(\mathrm{CH}_{2}\right), 28.2\left(\mathrm{CH}_{2}\right), 26.0$ $\left(\mathrm{CH}_{2}\right) \cdot$ Remark: one signal of a quaternary carbon is missing due to overlapping issue. HRMS (ESI/TOF) $m / z$ : $[\mathrm{M}+\mathrm{H}]^{+}$ Calcd for $\mathrm{C}_{23} \mathrm{H}_{21} \mathrm{~N}_{2} \mathrm{O}_{2}$ 357.1598; Found 357.1594. Crystals suited for X-Ray diffraction structure determination were obtained by slow evaporation of $\mathrm{CH}_{2} \mathrm{Cl}_{2}$ in $n$-heptane. CCDC 2049517 contains the crystallographic data for this compound.

5-Phenyl-9-(pyridin-3-yl)-1,6-diazabicyclo[4.3.0]nonane-

2,7-dione (7g). The title compound was prepared according to the above general procedure from $\mathbf{5 a}(52.9 \mathrm{mg}, 0.3 \mathrm{mmo}$, 1 equiv l) and nicotinaldehyde $3 \mathrm{~g}$ (28.2 $\mu \mathrm{L}, 0.3 \mathrm{mmol}, 1$ equiv) in 16 hours. Two flash column chromatographies over silica gel (PE/EtOAc 10:90 v/v to EtOAc/MeOH 95:5 $\mathrm{v} / \mathrm{v}$; then $\mathrm{CH}_{2} \mathrm{Cl}_{2} / \mathrm{MeOH} 95: 5 \mathrm{v} / \mathrm{v}$ ) were mandatory to afford the pure title compound as a yellow solid $(60.6 \mathrm{mg}$, $66 \%$, $>95: 5 d r) \cdot R_{f}=0.17\left(\mathrm{CH}_{2} \mathrm{Cl}_{2} / \mathrm{MeOH} 95: 5 \mathrm{v} / \mathrm{v}\right)$. m.p. $101-102$ ${ }^{\circ} \mathrm{C}$. IR (neat) $v_{\max } 2929,1695,1643,1349,1332,1298,1254$, $697,455 \mathrm{~cm}^{-1} .{ }^{1} \mathrm{H}$ NMR $(300 \mathrm{MHz}, \mathrm{CDCl} 3) \delta_{\mathrm{H}} 8.64(\mathrm{~d}, J=17.1$ $\mathrm{Hz}, 2 \mathrm{H}), 7.64(\mathrm{dt}, J=7.8,1.6 \mathrm{~Hz}, 2 \mathrm{H}), 7.33-7.28(\mathrm{~m}, 3 \mathrm{H})$, 7.19-7.15 (m, 2H), $5.86(\mathrm{dd}, J=10.7,3.1 \mathrm{~Hz}, 1 \mathrm{H}), 5.61(\mathrm{t}, J=$ $5.0 \mathrm{~Hz}, 1 \mathrm{H}$ ), 3.38 (dd, $J=17.5,10.7 \mathrm{~Hz}, 1 \mathrm{H}$ ), 2.89 (dd, $J=17.5$, $3.1 \mathrm{~Hz}, 1 \mathrm{H}), 2.56-2.52(\mathrm{~m}, 2 \mathrm{H}), 2.39-2.32(\mathrm{~m}, 2 \mathrm{H}) .{ }^{13} \mathrm{C}\left\{{ }^{1} \mathrm{H}\right\}$ NMR (75 MHz, CDCl $)$ ) $\delta$ c $165.4(\mathrm{C}), 165.0(\mathrm{C}), 149.7(\mathrm{CH})$, $148.1(\mathrm{CH}), 137.5(\mathrm{CH}), 134.7(\mathrm{C}), 134.0(\mathrm{CH}), 129.0(\mathrm{CH})$, $128.2(\mathrm{CH}), 125.7(\mathrm{CH}), 123.6(\mathrm{C}), 53.1(\mathrm{CH}), 52.4(\mathrm{CH}), 36.9$
$\left(\mathrm{CH}_{2}\right), 28.0\left(\mathrm{CH}_{2}\right), 25.7\left(\mathrm{CH}_{2}\right)$. HRMS (ESI/TOF) $\mathrm{m} / \mathrm{z}:[\mathrm{M}+\mathrm{H}]^{+}$ Calcd for $\mathrm{C}_{18} \mathrm{H}_{18} \mathrm{~N}_{3} \mathrm{O}_{2}$ 308.1394; Found 308.1402.

\section{5-Phenyl-9-(pyridin-4-yl)-1,6-diazabicyclo[4.3.0]nonane-}

2,7-dione (7h). The title compound was prepared according to the above general procedure from $5 \mathbf{a}(52.9 \mathrm{mg}, 0.3 \mathrm{mmol}$, 1 equiv) and isonicotinaldehyde $3 \mathbf{h}(28.3 \mu \mathrm{L}, 0.3 \mathrm{mmol}, 1$ equiv) in 16 hours. Two flash column chromatographies over silica gel (PE/EtOAc 10:90 v/v to EtOAc/MeOH 93:7 $\mathrm{v} / \mathrm{v}$; then $\mathrm{CH}_{2} \mathrm{Cl}_{2} / \mathrm{MeOH}$ 95:5 v/v) were mandatory to afford the title compound as a yellow solid (50.0 mg, 54\%, >95:5 $d r) . R_{f}=0.36\left(\mathrm{CH}_{2} \mathrm{Cl}_{2} / \mathrm{MeOH} 95: 5 \mathrm{v} / \mathrm{v}\right)$. m.p. $148-149^{\circ} \mathrm{C} . \mathrm{IR}$ (neat) $v_{\max } 2929,1701,1651,1333,1248,759,704,526$, $494 \mathrm{~cm}^{-1} .{ }^{1} \mathrm{H}$ NMR $\left(300 \mathrm{MHz}, \mathrm{CDCl}_{3}\right) \delta_{\mathrm{H}} 8.63(\mathrm{br} \mathrm{s}, 2 \mathrm{H}), 7.34-$ $7.28(\mathrm{~m}, 5 \mathrm{H}), 7.21-7.18(\mathrm{~m}, 2 \mathrm{H}), 5.82(\mathrm{dd}, J=10.9,3.2 \mathrm{~Hz}$ 1H), $5.63(\mathrm{t}, J=5.0 \mathrm{~Hz}, 1 \mathrm{H}), 3.39(\mathrm{dd}, J=17.5,10.9 \mathrm{~Hz}, 1 \mathrm{H})$, $2.85(\mathrm{dd}, J=17.5,3.2 \mathrm{~Hz}, 1 \mathrm{H}), 2.62-2.57(\mathrm{~m}, 2 \mathrm{H}), 2.50-2.30$ $(\mathrm{m}, 2 \mathrm{H}) \cdot{ }^{13} \mathrm{C}\left\{{ }^{1} \mathrm{H}\right\}$ NMR $\left(75 \mathrm{MHz}, \mathrm{CDCl}_{3}\right) \delta_{\mathrm{c}} 165.8(\mathrm{C}), 164.9$ (C), $150.6(\mathrm{CH}), 147.7(\mathrm{C}), 137.4(\mathrm{C}), 129.1(\mathrm{CH}), 128.4(\mathrm{CH})$, $125.9(\mathrm{CH}), 121.2(\mathrm{CH}), 54.1(\mathrm{CH}), 52.5(\mathrm{CH}), 36.8\left(\mathrm{CH}_{2}\right)$, $28.2\left(\mathrm{CH}_{2}\right), 25.7\left(\mathrm{CH}_{2}\right)$. HRMS (ESI/TOF) $\mathrm{m} / \mathrm{z}:[\mathrm{M}+\mathrm{H}]^{+}$Calcd for $\mathrm{C}_{18} \mathrm{H}_{18} \mathrm{~N}_{3} \mathrm{O}_{2}$ 308.1394; Found 308.1404.

9-Phenethyl-5-phenyl-1,6-diazabicyclo[4.3.0]nonane-2,7-dione (7i). The title compound was prepared according to the above general procedure from $\mathbf{5 a}(52.9 \mathrm{mg}, 0.3 \mathrm{mmol}, 1$ equiv) and hydrocinnamaldehyde $3 \mathbf{i}$ (39.5 $\mu \mathrm{L}, 0.3 \mathrm{mmol}, 1$ equiv) in 16 hours. Flash column chromatography over silica gel (PE/EtOAc 10:90 v/v to EtOAc/MeOH 98:2 v/v) afforded the title compound as a colourless oil $(81.4 \mathrm{mg}, 81 \%$, $>95: 5 d r$ ). $R_{f}=0.35$ (EtOAc/MeOH 98:2 v/v). IR (neat) $v_{\max }$ $2933,1698,1645,1453,1332,1255,750,698,559,496 \mathrm{~cm}^{-}$ ${ }^{1} .{ }^{1} \mathrm{H}$ NMR $\left(300 \mathrm{MHz}, \mathrm{CDCl}_{3}\right) \delta_{\mathrm{H}} 7.40-7.19(\mathrm{~m}, 10 \mathrm{H}), 5.55(\mathrm{t}, J$ 
$=5.1 \mathrm{~Hz}, 1 \mathrm{H}), 4.84(\mathrm{dtd}, J=9.6,7.2,2.2 \mathrm{~Hz}, 1 \mathrm{H}), 2.97(\mathrm{dd}, J=$ $17.2,9.6 \mathrm{~Hz}, 1 \mathrm{H}), 2.82-2.65(\mathrm{~m}, 2 \mathrm{H}), 2.50-2.19(\mathrm{~m}, 6 \mathrm{H})$, 1.95-1.82 (m, 1H). ${ }^{13} \mathrm{C}\left\{{ }^{1} \mathrm{H}\right\}$ NMR $\left(75 \mathrm{MHz}, \mathrm{CDCl}_{3}\right) \delta_{\mathrm{C}} 165.9$ (C), $165.6(\mathrm{C}), 140.7(\mathrm{C}), 138.1(\mathrm{C}), 129.0(\mathrm{CH}), 128.6(\mathrm{CH})$, $128.4(\mathrm{CH}), 128.1(\mathrm{CH}), 126.3(\mathrm{CH}), 125.6(\mathrm{CH}), 52.7(\mathrm{CH})$, $51.9(\mathrm{CH}), 36.2\left(\mathrm{CH}_{2}\right), 35.9\left(\mathrm{CH}_{2}\right), 32.0\left(\mathrm{CH}_{2}\right), 28.0\left(\mathrm{CH}_{2}\right)$, $26.2\left(\mathrm{CH}_{2}\right)$. HRMS (CI/TOF) $\mathrm{m} / \mathrm{z}:[\mathrm{M}+\mathrm{H}]^{+}$Calcd for $\mathrm{C}_{21} \mathrm{H}_{23} \mathrm{~N}_{2} \mathrm{O}_{2}$ 335.1754; Found 335.1758.

\section{9-Isobutyl-5-phenyl-1,6-diazabicyclo[4.3.0]nonane-2,7-di-}

one (7j). The title compound was prepared according to the above general procedure from $5 \mathbf{a}(52.9 \mathrm{mg}, 0.3 \mathrm{mmol}, 1$ equiv) and isovaleraldehyde $\mathbf{3 j}$ (32.2 $\mu \mathrm{L}, 0.3 \mathrm{mmol}, 1$ equiv $)$ in 30 hours. Flash column chromatography over silica gel (PE/EtOAc 10:90 v/v to EtOAc/MeOH 98:2 v/v) afforded the title compound as a yellowish solid (52.1 mg, 61\%, $>95: 5 d r) . R_{f}=0.57(\mathrm{EtOAc} / \mathrm{MeOH} 98: 2 \mathrm{v} / \mathrm{v})$. m.p. $85-86^{\circ} \mathrm{C}$. IR (neat) $v_{\max } 2950,1685,1325,1226,1167,702,606,536$ $\mathrm{cm}^{-1} .{ }^{1} \mathrm{H}$ NMR $\left(300 \mathrm{MHz}, \mathrm{CDCl}_{3}\right) \delta_{\mathrm{H}} 7.40-7.24(\mathrm{~m}, 5 \mathrm{H}), 5.52$ $(\mathrm{t}, J=5.2 \mathrm{~Hz}, 1 \mathrm{H}), 4.91-4.82(\mathrm{~m}, 1 \mathrm{H}), 2.95(\mathrm{dd}, J=17.1,9.4$ $\mathrm{Hz}, 1 \mathrm{H}), 2.46-2.25(\mathrm{~m}, 4 \mathrm{H}), 2.36(\mathrm{dd}, J=17.1,2.0 \mathrm{~Hz}, 1 \mathrm{H})$, 1.83 (ddd, $J=13.2,7.5,6.5 \mathrm{~Hz}, 1 \mathrm{H}$ ), 1.69 (hept, $J=6.7 \mathrm{~Hz}$, 1H), $1.38(\mathrm{dt}, J=13.2,7.5 \mathrm{~Hz}, 1 \mathrm{H}), 1.04(\mathrm{~d}, J=6.5 \mathrm{~Hz}, 3 \mathrm{H})$, $0.98(\mathrm{~d}, J=6.5 \mathrm{~Hz}, 3 \mathrm{H}) .{ }^{13} \mathrm{C}\left\{{ }^{1} \mathrm{H}\right\} \operatorname{NMR}\left(75 \mathrm{MHz}, \mathrm{CDCl}_{3}\right) \delta_{\mathrm{C}}$ $166.3(\mathrm{C}), 165.7$ (C), $138.3(\mathrm{C}), 129.0(\mathrm{CH}), 128.0(\mathrm{CH}), 125.7$ $(\mathrm{CH}), 52.0(\mathrm{CH}), 51.5(\mathrm{CH}), 43.3\left(\mathrm{CH}_{2}\right), 36.3\left(\mathrm{CH}_{2}\right), 28.1$ $\left(\mathrm{CH}_{2}\right), 26.5\left(\mathrm{CH}_{2}\right), 25.0(\mathrm{CH}), 22.6\left(\mathrm{CH}_{3}\right), 22.5\left(\mathrm{CH}_{3}\right) . \mathrm{HRMS}$ (CI/TOF) $m / z:[\mathrm{M}+\mathrm{H}]^{+}$Calcd for $\mathrm{C}_{17} \mathrm{H}_{23} \mathrm{~N}_{2} \mathrm{O}_{2}$ 287.1754; Found 287.1750.

5-Phenyl-9-isopropyl-1,6-diazabicyclo[4.3.0]nonane-2,7-dione (7k). The title compound was prepared according to the above general procedure from $\mathbf{5 a}(52.9 \mathrm{mg}, 0.3 \mathrm{mmol}, 1$ equiv) and isobutyraldehyde $3 \mathbf{k}(27.4 \mu \mathrm{L}, 0.3 \mathrm{mmol}, 1$ equiv) in 16 hours. Flash column chromatography over silica gel (PE/EtOAc 10:90 v/v to EtOAc/MeOH 98:2 v/v) afforded the title compound as a yellowish solid (68.4 mg, 84\%, >95:5 $d r) . R_{f}=0.32(\mathrm{EtOAc} / \mathrm{MeOH} 98: 2 \mathrm{v} / \mathrm{v})$. m.p. 94 ${ }^{\circ} \mathrm{C} . \mathrm{IR}$ (neat) $\nu_{\max } 2957,2917,2864,1700,1674,1333,1250$, $1181,768,700,527 \mathrm{~cm}^{-1} .{ }^{1} \mathrm{H}$ NMR $\left(300 \mathrm{MHz}, \mathrm{CDCl}_{3}\right) \delta_{\mathrm{H}} 7.40-$ $7.27(\mathrm{~m}, 5 \mathrm{H}), 5.49(\mathrm{t}, J=5.6 \mathrm{~Hz}, 1 \mathrm{H}), 4.51$ (ddd, $J=10.2,8.3$, $2.7 \mathrm{~Hz}, 1 \mathrm{H}), 2.84(\mathrm{dd}, J=17.4,10.2 \mathrm{~Hz}, 1 \mathrm{H}), 2.62-2.42(\mathrm{~m}$, $3 \mathrm{H}), 2.37-2.24(\mathrm{~m}, 2 \mathrm{H}), 2.12-2.00(\mathrm{~m}, 1 \mathrm{H}), 0.98(\mathrm{~d}, J=6.8$ $\mathrm{Hz}, 3 \mathrm{H}), 0.95(\mathrm{~d}, J=6.7 \mathrm{~Hz}, 3 \mathrm{H}) .{ }^{13} \mathrm{C}\left\{{ }^{1} \mathrm{H}\right\} \mathrm{NMR}(75 \mathrm{MHz}$, $\left.\mathrm{CDCl}_{3}\right) \delta_{\mathrm{C}} 167.3(\mathrm{C}), 166.4(\mathrm{C}), 138.2(\mathrm{C}), 128.9(\mathrm{CH}), 128.1$ $(\mathrm{CH}), 126.0(\mathrm{CH}), 58.5(\mathrm{CH}), 52.6(\mathrm{CH}), 33.4\left(\mathrm{CH}_{2}\right), 31.6(\mathrm{CH})$, $28.7\left(\mathrm{CH}_{2}\right), 26.3\left(\mathrm{CH}_{2}\right), 19.0\left(\mathrm{CH}_{3}\right), 17.7\left(\mathrm{CH}_{3}\right)$. HRMS (CI/TOF): $[\mathrm{M}+\mathrm{H}]^{+}$Calcd for $\mathrm{C}_{16} \mathrm{H}_{21} \mathrm{~N}_{2} \mathrm{O}_{2}$ 273.1598; Found 273.1594. Crystals suited for X-Ray diffraction structure determination were obtained by slow evaporation of $\mathrm{CH}_{2} \mathrm{Cl}_{2}$ in n-heptane. CCDC 2049519 contains the crystallographic data for this compound.

9-Cyclopropyl-5-phenyl-1,6-diazabicyclo[4.3.0]nonane-2,7dione (기). The title compound was prepared according to the above general procedure from $5 \mathbf{5 a}(52.9 \mathrm{mg}, 0.3 \mathrm{mmol}, 1$ equiv) and cyclopropanecarbaldehyde $3 \mathbf{l}(22.4 \mu \mathrm{L}, 0.3$ mmol, 1 equiv) in 24 hours. Flash column chromatography over silica gel (PE/EtOAc 10:90 v/v to EtOAc/MeOH 98:2 $\mathrm{v} / \mathrm{v}$ ) afforded the title compound as a colourless oil (55.3 $\mathrm{mg}, 68 \%,>95: 5 d r$ ). $R_{f}=0.17$ (EtOAc). IR (neat) $v_{\max } 3004$, $1698,1643,1348,1333,1244,1020,700 \mathrm{~cm}^{-1} .{ }^{1} \mathrm{H}$ NMR $(300$ $\left.\mathrm{MHz}_{\mathrm{CDCl}}\right) \delta_{\mathrm{H}} 7.40-7.28(\mathrm{~m}, 5 \mathrm{H}), 5.55(\mathrm{t}, J=4.9 \mathrm{~Hz}, 1 \mathrm{H})$, $4.35-4.28(\mathrm{~m}, 1 \mathrm{H}), 2.94(\mathrm{dd}, J=17.2,9.8 \mathrm{~Hz}, 1 \mathrm{H}), 2.52-2.42$ (m, 3H), 2.31-2.24 (m, 2H), 1.33-1.21 (m, 1H), 0.73-0.58 
(m, 3H), 0.40-0.34 (m, 1H). ${ }^{13} \mathrm{C}\left\{{ }^{1} \mathrm{H}\right\} \operatorname{NMR}\left(75 \mathrm{MHz}, \mathrm{CDCl}_{3}\right) \delta_{\mathrm{c}}$ $165.9(\mathrm{C}), 164.8(\mathrm{C}), 138.3(\mathrm{C}), 129.0(\mathrm{CH}), 128.0(\mathrm{CH}), 125.7$ $(\mathrm{CH}), 56.7(\mathrm{CH}), 51.9(\mathrm{CH}), 35.3\left(\mathrm{CH}_{2}\right), 27.8\left(\mathrm{CH}_{2}\right), 26.4$ $\left(\mathrm{CH}_{2}\right), 15.4(\mathrm{CH}), 3.8\left(\mathrm{CH}_{2}\right), 2.4\left(\mathrm{CH}_{2}\right)$. HRMS (CI/TOF) $\mathrm{m} / \mathrm{z}$ : $[\mathrm{M}+\mathrm{H}]^{+}$Calcd for $\mathrm{C}_{16} \mathrm{H}_{19} \mathrm{~N}_{2} \mathrm{O}_{2}$ 271.1441; Found 271.1454.

9-((Benzyloxy)methyl)-5-phenyl-1,6-diazabicyclo[4.3.0]nonane-2,7-dione $(7 \mathrm{~m})$. The title compound was prepared according to the above general procedure from $\mathbf{5 a}(52.9 \mathrm{mg}$, 0.3 mmol, 1 equiv) and (benzyloxy)acetaldehyde 3 m (42.2 $\mu \mathrm{L}, 0.3 \mathrm{mmol}, 1$ equiv) in 24 hours. Flash column chromatography over silica gel (PE/EtOAc 10:90 v/v to EtOAc/MeOH 95:5 v/v) afforded the title compound as a colourless oil $(38.4 \mathrm{mg}, 37 \%,>95: 5 d r) . \quad R_{f}=0.23$ (EtOAc/MeOH 95:5 v/v). IR (neat) $v_{\max } 3030,2924,2858$, $1699,1642,1354,1261,1115,1067,751,698 \mathrm{~cm}^{-1} .{ }^{1} \mathrm{H}$ NMR $\left(300 \mathrm{MHz}, \mathrm{CDCl}_{3}\right) \delta_{\mathrm{H}} 7.43-7.29(\mathrm{~m}, 7 \mathrm{H}), 7.23-7.09(\mathrm{~m}, 3 \mathrm{H})$, $5.60(\mathrm{t}, J=4.3 \mathrm{~Hz}, 1 \mathrm{H}), 4.92-4.85(\mathrm{~m}, 1 \mathrm{H}), 4.66(\mathrm{~d}, J=11.9$ $\mathrm{Hz}, 1 \mathrm{H}, \mathrm{AB}), 4.57(\mathrm{~d}, J=11.9 \mathrm{~Hz}, 1 \mathrm{H}, \mathrm{AB}), 3.96(\mathrm{dd}, J=9.7,3.5$ $\mathrm{Hz}, 1 \mathrm{H}), 3.60(\mathrm{dd}, J=9.7,3.5 \mathrm{~Hz}, 1 \mathrm{H}), 2.95(\mathrm{dd}, J=17.3,10.2$ $\mathrm{Hz}, 1 \mathrm{H}), 2.85(\mathrm{dd}, J=17.3,4.2 \mathrm{~Hz}, 1 \mathrm{H}), 2.48-2.17(\mathrm{~m}, 4 \mathrm{H})$. ${ }^{13} \mathrm{C}\left\{{ }^{1} \mathrm{H}\right\}$ NMR (75 MHz, $\left.\mathrm{CDCl}_{3}\right) \delta_{\mathrm{C}} 165.3(\mathrm{C}), 164.1(\mathrm{C}), 137.6$ (C), $137.5(\mathrm{C}), 128.8(\mathrm{CH}), 128.6(\mathrm{CH}), 128.0(\mathrm{CH}), 127.82$ $(\mathrm{CH}), 127.76(\mathrm{CH}), 125.7(\mathrm{CH}), 73.6\left(\mathrm{CH}_{2}\right), 69.9\left(\mathrm{CH}_{2}\right), 52.5$ $(\mathrm{CH}), 52.2(\mathrm{CH}), 32.4\left(\mathrm{CH}_{2}\right), 27.5\left(\mathrm{CH}_{2}\right), 25.8\left(\mathrm{CH}_{2}\right) . \mathrm{HRMS}$ (CI/TOF) m/z: [M+H] ${ }^{+}$Calcd for $\mathrm{C}_{21} \mathrm{H}_{23} \mathrm{~N}_{2} \mathrm{O}_{3}$ 351.1703; Found 351.1704.

5-Methyl-9-phenyl-1,6-diazabicyclo[4.3.0]nonane-2,7-dione

(7n). The title compound was prepared according to the above general procedure from 6-methyltetrahydropyridazin-3(2H)-one $\mathbf{5 b}$ (34.2 $\mathrm{mg}, 0.3 \mathrm{mmol}, 1$ equiv) and benzaldehyde $3 a$ ( $30.5 \mu \mathrm{L}, 0.3 \mathrm{mmol}, 1$ equiv) in 16 hours to give the crude product as a mixture of stereoisomers $(89 \%$ NMR yield, 82:18 $d r$ ). Flash column chromatography over silica gel (PE/EtOAc 10:90 v/v to EtOAc/MeOH 97:3 v/v) afforded the title compound as a diastereo-enriched mixture as a white solid $(34.7 \mathrm{mg}, 47 \%, 93: 7 d r) . R_{f}=0.27$ for the minor diastereoisomer, 0.20 for the major diastereoisomer (EtOAc/MeOH 97:3 v/v). IR (neat) $v_{\max } 1678,1641,1357$, $698 \mathrm{~cm}^{-1} .{ }^{1} \mathrm{H}$ NMR $\left(300 \mathrm{MHz}, \mathrm{CDCl}_{3}\right) \delta_{\mathrm{H}} 7.39-7.27(\mathrm{~m}, 5 \mathrm{H})$, 5.71 (dd, $J=10.8,2.7 \mathrm{~Hz}, 0.89 \mathrm{H}$, major diastereoisomer), $5.57(\mathrm{dd}, J=10.8,3.5 \mathrm{~Hz}, 0.05 \mathrm{H}$, minor diastereoisomer), $4.63(\mathrm{~h}, J=6.5 \mathrm{~Hz}, 1 \mathrm{H}), 3.26(\mathrm{dd}, J=17.2,10.6 \mathrm{~Hz}, 1 \mathrm{H}), 2.69$ $(\mathrm{dd}, J=17.2,2.7 \mathrm{~Hz}, 1 \mathrm{H}), 2.72-2.46(\mathrm{~m}, 2 \mathrm{H}), 2.17-2.05(\mathrm{~m}$, 1H), $1.91-1.81(\mathrm{~m}, 1 \mathrm{H}), 1.38(\mathrm{~d}, J=6.5 \mathrm{~Hz}, 3 \mathrm{H}) .{ }^{13} \mathrm{C}\left\{{ }^{1} \mathrm{H}\right\} \mathrm{NMR}$ $\left(75 \mathrm{MHz}, \mathrm{CDCl}_{3}\right) \delta_{\mathrm{C}} 164.8(\mathrm{C}), 164.6(\mathrm{C}), 139.9(\mathrm{C}), 129.1$ (CH), $128.3(\mathrm{CH}), 125.7(\mathrm{CH}), 55.0(\mathrm{CH}), 45.3(\mathrm{CH}), 38.4$ $\left(\mathrm{CH}_{2}\right), 27.8\left(\mathrm{CH}_{2}\right), 26.0\left(\mathrm{CH}_{2}\right), 17.1\left(\mathrm{CH}_{3}\right) . \mathrm{HRMS}(\mathrm{ESI} / \mathrm{TOF})$ $m / z: \quad[\mathrm{M}+\mathrm{H}]^{+}$Calcd for $\mathrm{C}_{14} \mathrm{H}_{17} \mathrm{~N}_{2} \mathrm{O}_{2}$ 245.1285; Found 245.1285.

\section{5-Ethyl-9-phenyl-1,6-diazabicyclo[4.3.0]nonane-2,7-dione}

(7o). The title compound was prepared according to the above general procedure from 6-ethyltetrahydropyridazin3(2H)-one 5c (38.5 mg, $0.3 \mathrm{mmol}, 1$ equiv) and benzaldehyde 3a (30.5 $\mu \mathrm{L}, 0.3 \mathrm{mmol}, 1$ equiv) in 16 hours to give the crude product as a mixture of stereoisomers (71\% NMR yield, 92:8 $d r$ ). Flash column chromatography over silica gel (PE/EtOAc 10:90 v/v to EtOAc/MeOH 97:3 v/v) afforded the major diastereoisomer as a yellow oil (42.6 mg, 55\%, $>95: 5 d r$ ). $R_{f}=0.39$ for the minor diastereoisomer, 0.24 for the major diastereoisomer (EtOAc/MeOH 97:3 v/v). IR (neat) $v_{\max } 1688,1639,1358,726 \mathrm{~cm}^{-1} .{ }^{1} \mathrm{H}$ NMR $(300 \mathrm{MHz}$, $\left.\mathrm{CDCl}_{3}\right) \delta_{\mathrm{H}} 7.39-7.27(\mathrm{~m}, 5 \mathrm{H}), 5.75(\mathrm{dd}, J=10.5,2.6 \mathrm{~Hz}, 1 \mathrm{H})$, 
4.45-4.37 (m, 1H), $3.26(\mathrm{dd}, J=17.2,10.5 \mathrm{~Hz}, 1 \mathrm{H}), 2.74(\mathrm{dd}$, $J=17.2,2.6 \mathrm{~Hz}, 1 \mathrm{H}), 2.67-2.46(\mathrm{~m}, 2 \mathrm{H}), 2.12-1.91(\mathrm{~m}, 2 \mathrm{H})$, 1.90-1.77 (m, 1H), 1.68-1.56 (m, 1H), $0.97(\mathrm{t}, J=7.4 \mathrm{~Hz}, 3 \mathrm{H})$. ${ }^{13} \mathrm{C}\left\{{ }^{1} \mathrm{H}\right\}$ NMR $\left(75 \mathrm{MHz}, \mathrm{CDCl}_{3}\right) \delta_{\mathrm{C}} 165.1(\mathrm{C}), 164.7(\mathrm{C}), 139.7$ (C), $129.1(\mathrm{CH}), 128.3(\mathrm{CH}), 125.8(\mathrm{CH}), 54.9(\mathrm{CH}), 50.8$ (CH), $38.2\left(\mathrm{CH}_{2}\right), 27.7\left(\mathrm{CH}_{2}\right), 24.0\left(\mathrm{CH}_{2}\right), 23.6\left(\mathrm{CH}_{2}\right), 10.5$ $\left(\mathrm{CH}_{3}\right)$. HRMS (ESI/TOF) $m / z$ : $[\mathrm{M}+\mathrm{H}]^{+}$Calcd for $\mathrm{C}_{15} \mathrm{H}_{19} \mathrm{~N}_{2} \mathrm{O}_{2}$ 259.1441; Found 259.1442.

\section{5-Allyl-9-phenyl-1,6-diazabicyclo[4.3.0]nonane-2,7-dione}

(7p). The title compound was prepared according to the above general procedure from 6-allyltetrahydropyridazin3(2H)-one $5 \mathbf{d}$ (42.1 mg, $0.3 \mathrm{mmol}, 1$ equiv) and benzaldehyde 3a (30.5 $\mu \mathrm{L}, 0.3 \mathrm{mmol}, 1$ equiv) in 16 hours to give the crude product as a mixture of stereoisomers $(88 \%$ NMR yield, 90:10 dr). Flash column chromatography over silica gel (PE/EtOAc 10:90 v/v to EtOAc/MeOH 97:3 v/v) afforded the major diastereoisomer as a yellow oil (51.9 mg, $64 \%,>95: 5 d r) . R_{f}=0.49$ for the minor diastereoisomer, 0.25 for the major diastereoisomer (EtOAc/MeOH 98:2 v/v). IR (neat) $v_{\max } 1693,1644,1353,1333,1256,698 \mathrm{~cm}^{-1}$ ${ }^{1} \mathrm{H}$ NMR $\left(300 \mathrm{MHz}^{\mathrm{CDCl}} 3\right) \delta_{\mathrm{H}} 7.38-7.28(\mathrm{~m}, 5 \mathrm{H}), 5.84-5.70$ (m, 1H), $5.72(\mathrm{dd}, J=10.6,2.9 \mathrm{~Hz}, 1 \mathrm{H}), 5.15-5.07(\mathrm{~m}, 2 \mathrm{H})$, $4.61-4.53(\mathrm{~m}, 1 \mathrm{H}), 3.25(\mathrm{dd}, J=17.3,10.6 \mathrm{~Hz}, 1 \mathrm{H}), 2.73(\mathrm{dd}$, $J=17.3,2.9 \mathrm{~Hz}, 1 \mathrm{H}), 2.67-2.46(\mathrm{~m}, 3 \mathrm{H}), 2.41-2.31(\mathrm{~m}, 1 \mathrm{H})$, 2.09-1.93 (m, 2H). ${ }^{13} \mathrm{C}\left\{{ }^{1} \mathrm{H}\right\} \mathrm{NMR}\left(75 \mathrm{MHz}, \mathrm{CDCl}_{3}\right) \delta_{\mathrm{C}} 164.9$ (C), $164.8(\mathrm{C}), 139.6(\mathrm{C}), 132.8(\mathrm{CH}), 129.1(\mathrm{CH}), 128.4(\mathrm{CH})$, $126.1(\mathrm{CH}), 119.2\left(\mathrm{CH}_{2}\right), 55.0(\mathrm{CH}), 48.7(\mathrm{CH}), 38.0\left(\mathrm{CH}_{2}\right)$, $35.4\left(\mathrm{CH}_{2}\right), 27.6\left(\mathrm{CH}_{2}\right), 23.4\left(\mathrm{CH}_{2}\right)$. HRMS (ESI/TOF) $m / z$ : $[\mathrm{M}+\mathrm{H}]^{+}$Calcd for $\mathrm{C}_{16} \mathrm{H}_{18} \mathrm{~N}_{2} \mathrm{O}_{2} 271.1441 ;$ Found 271.1443. 5-Methylcarboxy-9-phenyl-1,6-diazabicyclo[4.3.0]nonane2,7-dione (7q). The title compound was prepared according to the above general procedure from tetrahydropyridazinone 5e (47.4 mg, $0.3 \mathrm{mmol}, 1$ equiv) and benzaldehyde $\mathbf{3 a}$ ( $30.5 \mu \mathrm{L}, 0.3 \mathrm{mmol}, 1$ equiv) in 16 hours to give the crude product as a mixture of stereoisomers (81\% NMR yield, 85:15 dr). Flash column chromatography over silica gel (EtOAc/MeOH 98:2 v/v) afforded the major diastereoisomer $\mathbf{7 q}$ as a yellow oil $(67.3 \mathrm{mg}, 78 \%,>95: 5 d r)$. IR (neat) $v_{\max } 3488,2954,1743,1702,1647,1351,1258,1213,954$, $699 \mathrm{~cm}^{-1} .{ }^{1} \mathrm{H} \mathrm{NMR}\left(300 \mathrm{MHz}, \mathrm{CDCl}_{3}\right) \delta_{\mathrm{H}} 7.47-7.44(\mathrm{~m}, 2 \mathrm{H})$, $7.41-7.29(\mathrm{~m}, 3 \mathrm{H}), 5.67(\mathrm{dd}, J=10.8,3.4 \mathrm{~Hz}, 1 \mathrm{H}), 5.10(\mathrm{dd}, J$ = 5.7, $4.1 \mathrm{~Hz}, 1 \mathrm{H}), 3.79(\mathrm{~s}, 3 \mathrm{H}), 3.31(\mathrm{dd}, J=17.6,10.8 \mathrm{~Hz}$, 1H), $2.77(\mathrm{dd}, J=17.6,3.4 \mathrm{~Hz}, 1 \mathrm{H}), 2.55-2.41(\mathrm{~m}, 3 \mathrm{H}), 2.25-$ $2.13(\mathrm{~m}, 1 \mathrm{H}) .{ }^{13} \mathrm{C}\left\{{ }^{1} \mathrm{H}\right\} \mathrm{NMR}\left(75 \mathrm{MHz}, \mathrm{CDCl}_{3}\right) \delta_{\mathrm{C}} 168.8,165.4$, $163.4,139.9,129.0$ (2C), 128.3, 126.2 (2C), 55.6, 53.1, 51.3, 38.2, 28.5, 22.1. HRMS (API/TOF) $m / z$ : $[\mathrm{M}+\mathrm{H}]^{+}$Calcd for $\mathrm{C}_{15} \mathrm{H}_{17} \mathrm{~N}_{2} \mathrm{O}_{4}$ 289.1183; Found 289.1186.

\section{5-tert-Butylcarboxy-9-phenyl-1,6-diazabicyclo[4.3.0]non-}

ane-2,7-dione (7r). The title compound was prepared according to the above general procedure from tetrahydropyridazinone $\mathbf{5 f}$ (60.1 mg, $0.3 \mathrm{mmol}, 1$ equiv) and benzaldehyde $3 \mathbf{a}(30.5 \mu \mathrm{L}, 0.3 \mathrm{mmol}, 1$ equiv) in 16 hours to give the crude product as a mixture of stereoisomers (90\% NMR yield, 94:6 dr). Flash column chromatography over silica gel (EtOAc/MeOH 99:1 v/v) afforded the major diastereoisomer $7 \mathbf{r}$ as a yellow oil $(86.5 \mathrm{mg}, 87 \%,>95: 5 d r)$. IR (neat) $\nu_{\max } 2978,1733,1705,1651,1455,1352,1255,1150,842$, $698 \mathrm{~cm}^{-1} .{ }^{1} \mathrm{H}$ NMR $\left(300 \mathrm{MHz}, \mathrm{CDCl}_{3}\right) \delta_{\mathrm{H}} 7.50-7.47(\mathrm{~m}, 2 \mathrm{H})$, $7.41-7.28(\mathrm{~m}, 3 \mathrm{H}), 5.65(\mathrm{dd}, J=10.9,3.8 \mathrm{~Hz}, 1 \mathrm{H}), 4.98(\mathrm{dd}, J$ $=5.7,4.3 \mathrm{~Hz}, 1 \mathrm{H}), 3.30(\mathrm{dd}, J=17.6,10.9 \mathrm{~Hz}, 1 \mathrm{H}), 2.76(\mathrm{dd}, J$ $=17.6,3.8 \mathrm{~Hz}, 1 \mathrm{H}), 2.58-2.51(\mathrm{~m}, 2 \mathrm{H}), 2.47-2.38(\mathrm{~m}, 1 \mathrm{H})$, 2.21-2.08 (m, 1H), 1.49 (s, 9H). ${ }^{13} \mathrm{C}\left\{{ }^{1} \mathrm{H}\right\}$ NMR (75 MHz, 
$\left.\mathrm{CDCl}_{3}\right) \delta_{\mathrm{c}} 167.2,165.3,163.5,140.1,128.9(2 \mathrm{C}), 128.1,126.1$

(2C), 83.5, 55.5, 52.1, 38.3, 28.5, 28.0 (3C), 22.1. HRMS

(API/TOF) $m / z$ : $[\mathrm{M}+\mathrm{H}]^{+}$Calcd for $\mathrm{C}_{18} \mathrm{H}_{23} \mathrm{~N}_{2} \mathrm{O}_{4}$ 331.1652;

Found 331.1649.

5-Methyl-9-(naphthalen-2-yl)-1,6-diazabicyclo[4.3.0]non-

ane-2,7-dione (7s). The title compound was prepared according to the above general procedure from 6-methyltetrahydropyridazin-3(2H)-one $\mathbf{5 b}(34.2 \mathrm{mg}, 0.3 \mathrm{mmol}, 1$ equiv) and 2-naphthaldehyde $3 f(46.9 \mathrm{mg}, 0.3 \mathrm{mmol}, 1$ equiv) in 16 hours to give the crude product as a mixture of stereoisomers (100\% NMR yield, 85:15 dr). Flash column chromatography over silica gel (EtOAc to EtOAc/MeOH 97:3 $\mathrm{v} / \mathrm{v}$ ) afforded the title compound as a diastereo-enriched mixture as a yellow solid (44.2 mg, 57\%, 96:4 dr). $R_{f}=0.43$ for the minor diastereoisomer, 0.37 for the major diastereoisomer (EtOAc/MeOH 95:5 v/v). IR (neat) $v_{\max } 1673,1329$, $1179,822,756,474,408 \mathrm{~cm}^{-1} .{ }^{1} \mathrm{H}$ NMR $\left(300 \mathrm{MHz}, \mathrm{CDCl}_{3}\right) \delta_{\mathrm{H}}$ 7.87-7.75 (m, 4H), 7.52-7.46 (m, 2H), $7.38(\mathrm{dd}, J=8.5,1.9$ $\mathrm{Hz}, 1 \mathrm{H}), 5.87(\mathrm{dd}, J=10.5,2.7 \mathrm{~Hz}, 1 \mathrm{H}), 4.68(\mathrm{dq}, J=12.1,6.5$ $\mathrm{Hz}, 1 \mathrm{H}), 3.33(\mathrm{dd}, J=17.3,10.5 \mathrm{~Hz}, 1 \mathrm{H}), 2.79(\mathrm{dd}, J=17.3$, $2.7 \mathrm{~Hz}, 1 \mathrm{H}), 2.72-2.48(\mathrm{~m}, 2 \mathrm{H}), 2.14(\mathrm{ddt}, J=13.9,11.0,5.8$ $\mathrm{Hz}, 1 \mathrm{H}), 1.88$ (ddt, $J=13.9,5.8,4.6 \mathrm{~Hz}, 1 \mathrm{H}), 1.41(\mathrm{~d}, J=6.5$ $\mathrm{Hz}, 3 \mathrm{H}) \cdot{ }^{13} \mathrm{C}\left\{{ }^{1} \mathrm{H}\right\} \mathrm{NMR}\left(75 \mathrm{MHz}, \mathrm{CDCl}_{3}\right) \delta_{\mathrm{C}} 164.9(\mathrm{C}), 164.6$ (C), 137.0 (C), 133.2 (C), $133.1(\mathrm{C}), 129.4(\mathrm{CH}), 128.1(\mathrm{CH})$, $127.8(\mathrm{CH}), 126.6(\mathrm{CH}), 126.5(\mathrm{CH}), 124.9(\mathrm{CH}), 123.4(\mathrm{CH})$, $55.2(\mathrm{CH}), 45.4(\mathrm{CH}), 38.2\left(\mathrm{CH}_{2}\right), 27.8\left(\mathrm{CH}_{2}\right), 26.0\left(\mathrm{CH}_{2}\right), 17.1$ $\left(\mathrm{CH}_{3}\right)$. HRMS (ESI/TOF) $m / z$ : $[\mathrm{M}+\mathrm{H}]^{+}$Calcd for $\mathrm{C}_{18} \mathrm{H}_{19} \mathrm{~N}_{2} \mathrm{O}_{2}$ 295.1441; Found 295.1453. Crystals suited for X-Ray diffraction structure determination were obtained by slow evaporation of $\mathrm{CH}_{2} \mathrm{Cl}_{2}$ in $n$-heptane. CCDC 2049520 contains the crystallographic data for this compound.
5-Methyl-9-phenethyl-1,6-diazabicyclo[4.3.0]nonane-2,7-

dione (7t). The title compound was prepared according to the above general procedure from 6-methyltetrahydropyridazin-3(2H)-one $\mathbf{5 b}$ (34.2 $\mathrm{mg}, 0.3 \mathrm{mmol}, 1$ equiv) and hydrocinnamaldehyde $3 \mathbf{i}$ (39.5 $\mu \mathrm{L}, 0.3 \mathrm{mmol}, 1$ equiv) in 16 hours to give the crude product as a mixture of stereoisomers (40\% NMR yield, 82:18 $d r$ ). Flash column chromatography over silica gel (PE/EtOAc 10:90 v/v to EtOAc/MeOH $97: 3 \mathrm{v} / \mathrm{v}$ ) afforded the major diastereoisomer as a colourless oil (14.0 mg, 17\%, >95:5 dr). $R_{f}=0.32$ for the minor diastereoisomer, 0.24 for the major diastereoisomer (EtOAc/MeOH 97:3 v/v). IR (neat) $v_{\max } 1693,1639,1361$, 1338, $700 \mathrm{~cm}^{-1} .{ }^{1} \mathrm{H}$ NMR $\left(300 \mathrm{MHz}, \mathrm{CDCl}_{3}\right) \delta_{\mathrm{H}} 7.31-7.28(\mathrm{~m}$, 2H), 7.21-7.17 (m, 3H), $4.75(\mathrm{dtd}, J=9.1,6.7,2.2 \mathrm{~Hz}, 1 \mathrm{H})$, 4.58-4.48 (m, 1H), 2.90 (dd, $J=17.1,9.6 \mathrm{~Hz}, 1 \mathrm{H}), 2.68$ (dd, $J$ $=8.8,7.2 \mathrm{~Hz}, 2 \mathrm{H}), 2.63-2.54(\mathrm{~m}, 1 \mathrm{H}), 2.50-2.40(\mathrm{~m}, 1 \mathrm{H}), 2.34$ (dd, $J=17.1,2.2 \mathrm{~Hz}, 1 \mathrm{H}), 2.14-2.02(\mathrm{~m}, 2 \mathrm{H}), 1.88-1.71(\mathrm{~m}$, 2H), $1.29(\mathrm{~d}, J=6.7 \mathrm{~Hz}, 3 \mathrm{H}) \cdot{ }^{13} \mathrm{C}\left\{{ }^{1} \mathrm{H}\right\} \mathrm{NMR}\left(75 \mathrm{MHz}, \mathrm{CDCl}_{3}\right) \delta_{\mathrm{C}}$ $165.5(\mathrm{C}), 165.1(\mathrm{C}), 140.8(\mathrm{C}), 128.7(\mathrm{CH}), 128.4(\mathrm{CH}), 126.3$ $(\mathrm{CH}), 52.6(\mathrm{CH}), 45.1(\mathrm{CH}), 36.0\left(\mathrm{CH}_{2}\right), 35.7\left(\mathrm{CH}_{2}\right), 31.5$ $\left(\mathrm{CH}_{2}\right), 28.0\left(\mathrm{CH}_{2}\right), 26.2\left(\mathrm{CH}_{2}\right), 17.1\left(\mathrm{CH}_{3}\right) . \mathrm{HRMS}(\mathrm{ESI} / \mathrm{TOF})$ $m / z: \quad[\mathrm{M}+\mathrm{H}]^{+}$Calcd for $\mathrm{C}_{16} \mathrm{H}_{21} \mathrm{~N}_{2} \mathrm{O}_{2}$ 273.1598; Found 273.1603.

\section{5-Methylcarboxy-9-phenethyl-1,6-diazabicyclo[4.3.0]non-}

ane-2,7-dione (7u). The title compound was prepared according to the above general procedure from tetrahydropyridazinone 5 e (47.4 mg, $0.3 \mathrm{mmol}, 1$ equiv) and hydrocinnamaldehyde $3 \mathbf{i}$ (39.5 $\mu \mathrm{L}, 0.3 \mathrm{mmol}, 1$ equiv) in 16 hours to give the crude product as a mixture of stereoisomers $196 \%$ NMR yield, >95:5 dr). Flash column chromatography over silica gel (EtOAc/MeOH 98:2 v/v) afforded the major 
diastereoisomer $\mathbf{7 u}$ as a white solid (82 mg, 86\%, >95:5 dr). m.p. $97-98^{\circ} \mathrm{C}$. IR (neat) $v_{\max } 2915,1742,1693,1631,1432$, 1370, 1240, 979, 757, $707 \mathrm{~cm}^{-1} .{ }^{1} \mathrm{H}$ NMR (300 MHz, $\left.\mathrm{CDCl}_{3}\right)$ $\delta_{\mathrm{H}} 7.32-7.17(\mathrm{~m}, 5 \mathrm{H}), 5.00(\mathrm{dd}, J=6.0,4.9 \mathrm{~Hz}, 1 \mathrm{H}), 4.80-4.72$ (m, 1H), $3.79(\mathrm{~s}, 3 \mathrm{H}), 2.94(\mathrm{dd}, J=17.2,9.6 \mathrm{~Hz}, 1 \mathrm{H}), 2.78-$ $2.64(\mathrm{~m}, 2 \mathrm{H}), 2.52-2.13(\mathrm{~m}, 6 \mathrm{H}), 1.97-1.85(\mathrm{~m}, 1 \mathrm{H}) .{ }^{13} \mathrm{C}\left\{{ }^{1} \mathrm{H}\right\}$ NMR (75 MHz, $\left.\mathrm{CDCl}_{3}\right) \delta_{\mathrm{C}} 168.8,166.0,164.1,140.8,128.5$ (2C), 128.4 (2C), 126.1, 53.1, 50.8, 35.42, 35.38, 31.4, 28.6, 22.5. HRMS (ESI/TOF) $m / z$ : $[\mathrm{M}+\mathrm{H}]^{+}$Calcd for $\mathrm{C}_{17} \mathrm{H}_{21} \mathrm{~N}_{2} \mathrm{O}_{4}$ 317.1496; Found 317.1492.

General procedure for the synthesis of 15, 17, 19 and 20. 1,6-Diazabicyclo[4.3.0]nonane-2,7-dione ( $\mathbf{\pm})-7 a(61.2 \mathrm{mg}$, $0.2 \mathrm{mmol}, 1$ equiv) was dissolved in THF (2 mL, $0.067 \mathrm{M})$ under nitrogen atmosphere. The solution was cooled down to $-78{ }^{\circ} \mathrm{C}$ (acetone/dry ice bath) and LiHMDS (273 $\mu \mathrm{L}, 2.2$ equiv, 1.6 $\mathrm{M}$ in THF) was added dropwise. After stirring at $78^{\circ} \mathrm{C}$ for 1 hour, a solution of electrophile $\mathrm{E}(0.3 \mathrm{mmol}, 1.5$ equiv) in THF (1 mL, $0.3 \mathrm{M}$ ) was added dropwise. The mixture was stirred at $-78{ }^{\circ} \mathrm{C}$ for 1 hour, then slow return to 10 ${ }^{\circ} \mathrm{C}$ was initiated by removing dry ice of the bath and the mixture was stirred for an additional 16 hours. The reaction was eventually quenched with a saturated aqueous $\mathrm{NH}_{4} \mathrm{Cl}$ solution at $0{ }^{\circ} \mathrm{C}$ and extracted three times with $\mathrm{CH}_{2} \mathrm{Cl}_{2}$. The combined organic layers were dried over $\mathrm{Na}_{2} \mathrm{SO}_{4}$, filtered and concentrated under reduced pressure. The crude product was then purified by flash column chromatography over silica gel to afford the product of electrophilic addition in 8 position (major product) which was in some cases obtained in an inseparable mixture with the product of addition in 3 position.
8-Methyl-5,9-diphenyl-1,6-diazabicyclo[4.3.0]nonane-2,7-

dione (15). The title compound was prepared according to the above general procedure from 1,6-diazabicyclo[4.3.0]nonane-2,7-dione 7a and methyl iodide $(18.7 \mu \mathrm{L}$, $0.3 \mathrm{mmol}, 1.5$ equiv) in 16 hours to give the crude product as a mixture of stereoisomers (84\% NMR yield, 85:15 $d r$ ). Flash column chromatography over silica gel (PE/EtOAc 30:70 v/v) afforded the major diastereoisomer 15 (mixture with 16 in a 94:6 ratio) as a colourless oil (49.2 mg, 77\%, $>95: 5 d r$ ). IR (neat) $v_{\max } 1695,1650,1329,1249,696 \mathrm{~cm}^{-1}$. ${ }^{1} \mathrm{H}$ NMR $\left(300 \mathrm{MHz}, \mathrm{CDCl}_{3}\right) \delta_{\mathrm{H}} 7.33-7.18(\mathrm{~m}, 8 \mathrm{H}), 7.17-7.10$ (m, 2H), 5.54-5.51 (m, 1H), $5.23(\mathrm{~d}, J=3.7 \mathrm{~Hz}, 1 \mathrm{H}), 2.91$ (ddd, $J=14.8,7.4,3.8 \mathrm{~Hz}, 1 \mathrm{H}), 2.46-2.40(\mathrm{~m}, 2 \mathrm{H}), 2.32-2.19$ (m, 2H), $1.40(\mathrm{~d}, J=7.4 \mathrm{~Hz}, 3 \mathrm{H}) \cdot{ }^{13} \mathrm{C}\left\{{ }^{1} \mathrm{H}\right\}$ NMR $(75 \mathrm{MHz}$, $\left.\mathrm{CDCl}_{3}\right) \delta_{\mathrm{C}} 168.7,165.5,139.0,137.8,129.0(2 \mathrm{C}), 128.9(2 \mathrm{C})$ 128.4, 128.1, 126.4 (2C), 126.0 (2C), 63.5, 52.3, 44.4, 28.0, 26.0, 16.5. HRMS (ESI/TOF) $m / z:[\mathrm{M}+\mathrm{H}]^{+}$Calcd for $\mathrm{C}_{20} \mathrm{H}_{21} \mathrm{~N}_{2} \mathrm{O}_{2}$ 321.1598; Found 321.1602.

5,9-Diphenyl-8-propargyl-1,6-diazabicyclo[4.3.0]nonane-

2,7-dione (17). The title compound was prepared according to the above general procedure from 1,6-diazabicyclo[4.3.0]nonane-2,7-dione 7a and propargyl bromide (33.4 $\mu \mathrm{L}, 0.3 \mathrm{mmol}, 1.5$ equiv, sol. $80 \% \mathrm{w} / \mathrm{w}$ in $\mathrm{PhMe}$ ) in 16 hours. Flash column chromatography over silica gel $\left(\mathrm{CH}_{2} \mathrm{Cl}_{2} / \mathrm{Et}_{2} \mathrm{O} 96: 4 \mathrm{v} / \mathrm{v}\right)$ afforded 17 (mixture with 18 in a 92:8 ratio) as a white solid (53.5 mg, 78\%, >95:5 dr). m.p. $115-116{ }^{\circ} \mathrm{C} . \mathrm{IR}$ (neat) $v_{\max } 3240,1700,1651,1493,1454$, $1349,1325,1257,750,697 \mathrm{~cm}^{-1} \cdot{ }^{1} \mathrm{H}$ NMR (300 MHz, $\left.\mathrm{CDCl}_{3}\right)$ $\delta_{\mathrm{H}} 7.41-7.25(\mathrm{~m}, 10 \mathrm{H}), 5.71(\mathrm{~d}, J=3.1 \mathrm{~Hz}, 1 \mathrm{H}), 5.66-5.63(\mathrm{~m}$, 1H), 3.11-3.06 (m, 1H), 2.89-2.73 (m, 2H), 2.56-2.51 (m, 2H), $2.40-2.33(\mathrm{~m}, 2 \mathrm{H}), 2.10(\mathrm{t}, J=2.6 \mathrm{~Hz}, 1 \mathrm{H}) .{ }^{13} \mathrm{C}\left\{{ }^{1} \mathrm{H}\right\} \mathrm{NMR}$ 
$\left(75 \mathrm{MHz} \mathrm{CDCl}_{3}\right) \delta_{\mathrm{c}} 166.3,165.8,139.1,137.7,129.1$ (2C), 129.0 (2C), 128.4, 128.2, 126.3 (2C), 126.1 (2C), 79.3, 71.3, 60.4, 52.7, 48.2, 28.3, 26.2, 20.9. HRMS (ESI/TOF) $\mathrm{m} / \mathrm{z}$ : $[\mathrm{M}+\mathrm{H}]^{+}$Calcd for $\mathrm{C}_{22} \mathrm{H}_{21} \mathrm{~N}_{2} \mathrm{O}_{2}$ 345.1598; Found 345.1595.

\section{8-Fluoro-5,9-diphenyl-1,6-diazabicyclo[4.3.0]nonane-2,7-}

dione (19). The title compound was prepared according to the above general procedure from 1,6-diazabicyclo[4.3.0]nonane-2,7-dione 7a (30.6 mg, $0.1 \mathrm{mmol}, 1$ equiv) and NFSI (63.1 mg, 2 equiv, $0.2 \mathrm{mmol}$ ) in 4 hours. Flash column chromatography over silica gel (PE/EtOAc 70:30 v/v to $60: 40 \mathrm{v} / \mathrm{v}$ ) afforded 19 as a white solid (17.5 mg, 54\%, $>95: 5 d r$ ). m.p. $104-105^{\circ} \mathrm{C}$. IR (neat) $v_{\max } 1727,1686,1322$, $1173,758,725,702 \mathrm{~cm}^{-1} .{ }^{1} \mathrm{H}$ NMR $\left(300 \mathrm{MHz}, \mathrm{CDCl}_{3}\right) \delta_{\mathrm{H}} 7.38$ $7.31(\mathrm{~m}, 6 \mathrm{H}), 7.27-7.22(\mathrm{~m}, 4 \mathrm{H}), 5.81\left(\mathrm{~d}, J_{\mathrm{HF}}=27.6 \mathrm{~Hz}, 1 \mathrm{H}\right)$, $5.62(\mathrm{t}, J=5.4 \mathrm{~Hz}, 1 \mathrm{H}), 5.19\left(\mathrm{dd}, J_{\mathrm{HF}}=51.7, J_{\mathrm{HH}}=1.3 \mathrm{~Hz}, 1 \mathrm{H}\right)$ 2.72-2.58 (m, 2H), 2.55-2.41 (m, 2H). ${ }^{13} \mathrm{C}\left\{{ }^{1} \mathrm{H}\right\}$ NMR (75 $\left.\mathrm{MHz}, \mathrm{CDCl}_{3}\right) \delta_{\mathrm{C}} 166.6(\mathrm{C}), 160.2(\mathrm{C})\left(\mathrm{d}, J_{\mathrm{CF}}=21.5 \mathrm{~Hz}\right), 137.0$ (C), $134.5(\mathrm{C})(\mathrm{d}, J=10.7 \mathrm{~Hz}), 129.4(\mathrm{CH}), 129.2(\mathrm{CH}), 129.1$ $(\mathrm{CH}), 128.6(\mathrm{CH}), 126.4(\mathrm{CH}), 126.2(\mathrm{CH}), 92.2(\mathrm{CH})\left(\mathrm{d}, J_{\mathrm{CF}}=\right.$ 191.5 Hz), $62.0(\mathrm{CH})\left(\mathrm{d}, J_{\mathrm{CF}}=23.8 \mathrm{~Hz}\right), 53.4(\mathrm{CH}), 28.5\left(\mathrm{CH}_{2}\right)$, $26.2\left(\mathrm{CH}_{2}\right)$. HRMS (ESI/TOF) $\mathrm{m} / \mathrm{z}:[\mathrm{M}+\mathrm{H}]^{+}$Calcd for $\mathrm{C}_{19} \mathrm{H}_{18} \mathrm{FN}_{2} \mathrm{O}_{2}$ 325.1347; Found 325.1353. Crystals suited for X-Ray diffraction structure determination were obtained by slow evaporation of $\mathrm{CH}_{2} \mathrm{Cl}_{2}$ in $n$-heptane. CCDC 2049518 contains the crystallographic data for this compound.

\section{8-Benzyl-5,9-diphenyl-1,6-diazabicyclo[4.3.0]nonane-2,7-}

dione (20). The title compound was prepared according to the above general procedure from 1,6-diazabicyclo[4.3.0]nonane-2,7-dione $\mathbf{7 a}$ and benzyl bromide (35.7 $\mu \mathrm{L}, 0.3 \mathrm{mmol}, 1.5$ equiv) in 16 hours. Flash column chromatography over silica gel (PE/EtOAc 60:40 v/v) afforded the major diastereoisomer 20 as a white solid (59.3 mg, 75\%, $>95: 5 d r$ ). m.p. $133-134{ }^{\circ}$ C. IR (neat) $\nu_{\max } 1692,1651,1454$, 1364, 1260, 886, 751, 731, 700, $669 \mathrm{~cm}^{-1} .{ }^{1} \mathrm{H}$ NMR $(300$ $\left.\mathrm{MHz}, \mathrm{CDCl}_{3}\right) \delta_{\mathrm{H}} 7.40-7.24(\mathrm{~m}, 11 \mathrm{H}), 7.20-7.12(\mathrm{~m}, 4 \mathrm{H}), 5.62$ $(\mathrm{d}, J=2.0 \mathrm{~Hz}, 1 \mathrm{H}), 5.46-5.43(\mathrm{~m}, 1 \mathrm{H}), 3.28-3.23(\mathrm{~m}, 1 \mathrm{H})$ $3.22-3.18(\mathrm{~m}, 2 \mathrm{H}), 2.35-2.23(\mathrm{~m}, 1 \mathrm{H}), 2.19-2.03(\mathrm{~m}, 2 \mathrm{H})$, 1.82-1.70 (m, 1H). ${ }^{13} \mathrm{C}\left\{{ }^{1} \mathrm{H}\right\}$ NMR $\left(75 \mathrm{MHz}, \mathrm{CDCl}_{3}\right) \delta_{\mathrm{C}} 167.3$, 164.7, 138.9, 137.7, 136.3, 129.7 (2C), 128.9 (2C), 128.84 (2C), 128.80 (2C), 128.2, 128.0, 127.4, 126.2 (2C), 126.0 (2C), 59.6, 52.1, 50.7, 36.8, 27.5, 25.5. HRMS (ESI/TOF) $m / z$ : $[\mathrm{M}+\mathrm{H}]^{+}$Calcd for $\mathrm{C}_{26} \mathrm{H}_{25} \mathrm{~N}_{2} \mathrm{O}_{2}$ 397.1911; Found 397.1920.

\section{ASSOCIATED CONTENT}

\section{Supporting Information}

The Supporting Information is available free of charge on the ACS Publications website

Full experimental details, spectral characterization and crystallographic data.

\section{AUTHOR INFORMATION}

\section{Corresponding Author}

Jean-François Brière - Normandie Univ, UNIROUEN, INSA Rouen, CNRS, COBRA, 76000 Rouen, France; orcid.org/00000002-1381-4535; E-mail: jean-francois.briere@insa-rouen.fr.

Corinne Loutelier-Bourhis - Normandie Univ, UNIROUEN, INSA Rouen, CNRS, COBRA (mass spectrometry department), 76000 Rouen, France; orcid.org/0000-0002-3361-1532; Email: corinne.loutelier@univ-rouen.fr.

\section{Author}

Arthur Lebrêne, Thomas Martzel, Laura Gouriou, Vincent Levacher, Sylvain Oudeyer, Carlos Afonso - Normandie Univ, UNIROUEN, INSA Rouen, CNRS, COBRA, 76000 Rouen, France. 
Morgane Sanselme - Laboratoire SMS - EA3233, Normandie

Univ-University of Rouen, France.

\section{Author Contributions}

tResearcher of the Mass spectroscopy department.

\section{Notes}

The authors declare no competing financial interest.

\section{ACKNOWLEDGMENT}

This work has been partially supported by INSA Rouen Normandy, University of Rouen Normandy, the Centre National de la Recherche Scientifique (CNRS), EFRD, and Labex SynOrg (ANR-11-LABX-0029), and by Region Normandie (CRUNCh network). This research was also supported by the French National Research Agency (ANR) as part of the ANR-16-CE070011-01 project OMaChem.

\section{REFERENCES}

(1) Blakemore, D. C.; Castro, L.; Churcher, I.; Rees, D. C.; Thomas, A. W.; Wilson, D. M.; Wood, A., Organic synthesis provides opportunities to transform drug discovery. Nat. Chem. 2018, 10, 383-394.

(2) (a) Murray, C. W.; Rees, D. C., Opportunity Knocks: Organic Chemistry for Fragment-Based Drug Discovery (FBDD). Angew. Chem. Int. Ed. 2016, 55, 488-492; (b) Kidd, S. L.; Osberger, T. J.; Mateu, N.; Sore, H. F.; Spring, D. R., Recent Applications of DiversityOriented Synthesis Toward Novel, 3-Dimensional Fragment Collections. Front. Chem. 2018, 6, 460; (c) Erlanson, D. A.; de Esch, I. J. P.; Jahnke, W.; Johnson, C. N.; Mortenson, P. N., Fragment-toLead Medicinal Chemistry Publications in 2018. J. Med. Chem. 2020, 63, 4430-4444 and references cited therein.

(3) Lovering, F., Escape from Flatland 2: complexity and promiscuity. MedChemComm 2013, 4, 515-519.

(4) (a) Qiu, G.; Kuang, Y.; Wu, J., N-Imide Ylide-Based Reactions: $\mathrm{C}-\mathrm{H}$ Functionalization, Nucleophilic Addition and Cycloaddition. Adv. Synth. Catal. 2014, 356, 3483-3504; (b) Najera, C.; Sansano, J. M.; Yus, M., 1,3-Dipolar cycloadditions of azomethine imines. Org. Biomol. Chem. 2015, 13, 8596-8636; (c) Svete, J.; Grošelj, U.; Požgan, F.; Štefane, B., Copper-Catalyzed Azomethine ImineAlkyne Cycloadditions (CuAIAC). Synthesis 2018, 50, 4501-4524.

(5) For selected organocatalyzed $(3+3)$ annulations of chiral azomethine imines, see: (a) Chan, A.; Scheidt, K. A., Highly stereoselective formal [3+3] cycloaddition of enals and azomethine imines catalyzed by $\mathrm{N}$-heterocyclic carbenes. J. Am. Chem. Soc. 2007, 129, 5334-5335; (b) Zhu, G.; Sun, W.; Wu, C.; Li, G.; Hong, L.; Wang, R., Base-Catalyzed Diastereoselective [3 + 3] Annulation of 3-Isothiocyanatooxindoles and Azomethine Imines. Org. Lett. 2013, 15, 4988-4991.

(6) For selected metal-catalyzed $(3+3)$ annulation with chiral azomethine imines, see: (a) Shintani, R.; Hayashi, T., Palladiumcatalyzed [3+3] cycloaddition of trimethylenemethane with azomethine imines. J. Am. Chem. Soc. 2006, 128, 6330-6331; (b)
Shapiro, N. D.; Shi, Y.; Toste, F. D., Gold-catalyzed [3+3]-annulation of azomethine imines with propargyl esters. J. Am. Chem. Soc. 2009, 131, 11654-11655; (c) Guo, H.; Liu, H.; Zhu, F.-L.; Na, R.; Jiang, H.; Wu, Y.; Zhang, L.; Li, Z.; Yu, H.; Wang, B.; Xiao, Y.; Hu, X.-P.; Wang, M., Enantioselective Copper-Catalyzed [3+3] Cycloaddition of Azomethine Ylides with Azomethine Imines. Angew. Chem. Int. Ed. 2013, 52, 12641-12645; (d) Tong, M.-C.; Chen, X.; Tao, H.-Y.; Wang, C.-J., Catalytic Asymmetric 1,3-Dipolar Cycloaddition of Two Different Ylides: Facile Access to Chiral 1,2,4-Triazinane Frameworks. Angew. Chem. Int. Ed. 2013, 52, 12377-12380; (e) Qian, Y.; Zavalij, P. J.; Hu, W.; Doyle, M. P., Bicyclic pyrazolidinone derivatives from diastereoselective catalytic [3+3]-cycloaddition reactions of enoldiazoacetates with azomethine imines. Org. Lett. 2013, 15, 1564-1567.

(7) (a) Roussi, F.; Bonin, M.; Chiaroni, A.; Micouin, L.; Riche, C.; Husson, H.-P., Asymmetric 1,3-dipolar cycloadditions of a chiral non-racemic azomethine imine. Tetrahedron Lett. 1999, 40, 37273730; (b) Roussi, F.; Chauveau, A.; Bonin, M.; Micouin, L.; Husson, H.-P., Diastereoselective Cycloadditions of Chiral Non-racemic Azomethine Imines. Synthesis 2000, 1170-1179; (c) Chung, F.; Chauveau, A.; Seltki, M.; Bonin, M.; Micouin, L., Asymmetric 1,3dipolar cycloadditions of a chiral nonracemic glyoxylic azomethine imine. Tetrahedron Lett. 2004, 45, 3127-3130.

(8) (a) Pair, E.; Berini, C.; Noël, R.; Sanselme, M.; Levacher, V.; Brière, J.-F., Organocatalysed multicomponent synthesis of pyrazolidinones: Meldrum's acid approach. Chem. Commun. 2014 50, 10218-10221; (b) Berini, C.; Sebban, M.; Oulyadi, H.; Sanselme, M.; Levacher, V.; Brière, J.-F., Organocatalyzed Multicomponent Synthesis of Isoxazolidin-5-ones. Org. Lett. 2015, 17, 5408-5411; (c) Martzel, T.; Annibaletto, J.; Millet, P.; Pair, E.; Sanselme, M.; Oudeyer, S.; Levacher, V.; Brière, J.-F., Organocatalytic Multicomponent Synthesis of alpha/beta-Dipeptide Derivatives. Chem.-Eur. J. 2020, 26, 8541-8545.

(9) (a) Dumas, A. M.; Fillion, E., Meldrum's Acids and 5Alkylidene Meldrum's Acids in Catalytic Carbon-Carbon BondForming Processes. Acc. Chem. Res. 2010, 43, 440-454; (b) Pair, E.; Cadart, T.; Levacher, V.; Brière, J.-F., Meldrum's Acid: a Useful Platform in Asymmetric Organocatalysis. ChemCatChem 2016, 8, 1882-1890.

(10) (a) Testa, E.; Fontanella, L., 1,6-Diazabicyclo[4.3.0]nonane2,7-diones and derivatives. Farmaco, Ed. Sci. 1971, 26, 1017-1035; (b) Tamura, N.; Matsushita, Y.; Yoshioka, K.; Ochiai, M., Synthesis of lactivicin analogues. Tetrahedron 1988, 44, 3231-3240.

(11) Gerencsér, J.; Dormán, G.; Darvas, F., Meldrum's Acid in Multicomponent Reactions: Applications to Combinatorial and Diversity-Oriented Synthesis. QSAR Comb. Sci. 2006, 25, 439-448.

(12) Evans, R. C.; Wiselogle, F. Y., Studies in the Pyridazine Series. The Absorption Spectrum of Pyridazine1. J. Am. Chem. Soc. 1945, 67, 60-62.

(13) For mechanistic investigation of Meldrum's acid based reaction by means of MS, see: (a) Montes D'Oca, C. R.; da Silva, J. C.; Guedes, E. P.; Montes D'Oca, M. G.; Santos, L. S.; Nachtigall, F. M.; Russowsky, D., A new multicomponent reaction for direct synthesis of primary $\gamma$-nitroamides. RSC Adv. 2016, 6, 98427 98433; (b) Lespes, N.; Pair, E.; Maganga, C.; Bretier, M.; Tognetti, V.; Joubert, L.; Levacher, V.; Hubert-Roux, M.; Afonso, C.; LoutelierBourhis, C.; Brière, J.-F., A Unique $(3+2)$ Annulation Reaction between Meldrum's Acid and Nitrones: Mechanistic Insight by ESIIMS-MS and DFT Studies. Chem. Eur. J. 2018, 24, 4086-4093.

(14) Paglia, G.; Williams, J. P.; Menikarachchi, L.; Thompson, J. W.; Tyldesley-Worster, R.; Halldórsson, S.; Rolfsson, O.; Moseley, A.; Grant, D.; Langridge, J.; Palsson, B. 0.; Astarita, G., Ion Mobility Derived Collision Cross Sections to Support Metabolomics Applications. Anal. Chem. 2014, 86, 3985-3993.

(15) Smith, D. P.; Knapman, T. W.; Campuzano, I.; Malham, R. W.; Berryman, J. T.; Radford, S. E.; Ashcroft, A. E., Deciphering drift time measurements from travelling wave ion mobility spectrometrymass spectrometry studies. Eur. J. Mass Spectrom. 2009, 15, 113130. 
(16) Kaumanns, 0.; Mayr, H., Electrophilicity Parameters of 5Benzylidene-2,2-dimethyl[1,3]dioxane-4,6-diones (Benzylidene Meldrum's Acids). J. Org. Chem. 2008, 73, 2738-2745.

(17) Hazard, R.; Tallec, A.; Tardivel, R.; Bourguignon, J. J.; Wermuth, C. G., Some observations on the electrochemical reduction of pyridazin-3-ones. Electrochim. Acta 1990, 35, 1907 1911.

(18) Banerjee, A.; Patil, S.; Pawar, M. Y.; Gullapalli, S.; Gupta, P. K.; Gandhi, M. N.; Bhateja, D. K.; Bajpai, M.; Sangana, R. R.; Gudi, G. S.; Khairatkar-Joshi, N.; Gharat, L. A., Imidazopyridazinones as novel PDE7 inhibitors: SAR and in vivo studies in Parkinson's disease model. Bioorg. Med. Chem. Lett. 2012, 22, 6286-6291.
(19) Taylor, E. C.; Hinkle, J. S., New routes to 1,2-diazetidin-3ones. J. Org. Chem. 1987, 52, 4107-4110.

(20) Katrusiak, A.; Piechowiak, P.; Katrusiak, A., Synthesis, tautomeric forms, specific intermolecular interactions, and lipophilicity of methylated 6-hydroxypyridazine-3-carboxylic acid and its 4,5-dihydro analogs. J. Mol. Struct. 2011, 998, 84-90.

(21) Alvarez-Ibarra, C.; Csákÿ, A. G.; Gómez de la Oliva, C., Diastereoselective Synthesis and Estimation of the Conformational Flexibility of 6-0xoperhydropyridazine-3-carboxylic Acid Derivatives. J. Org. Chem. 2002, 67, 2789-2797. 